MATHEMATICS OF COMPUTATION

Volume 80, Number 273, January 2011, Pages 593-615

S $0025-5718(2010) 02376-2$

Article electronically published on June 9, 2010

\title{
TORSION UNITS IN INTEGRAL GROUP RINGS OF JANKO SIMPLE GROUPS
}

\author{
V. A. BOVDI, E. JESPERS, AND A. B. KONOVALOV \\ Dedicated to the memory of Professor I. S. Luthar
}

\begin{abstract}
Using the Luthar-Passi method, we investigate the classical Zassenhaus conjecture for the normalized unit group of integral group rings of Janko sporadic simple groups. As a consequence, we obtain that the GruenbergKegel graph of the Janko groups $J_{1}, J_{2}$ and $J_{3}$ is the same as that of the normalized unit group of their respective integral group ring.
\end{abstract}

\section{Introduction AND MAIN RESUlts}

Let $V(\mathbb{Z} G)$ be the normalized unit group of the integral group ring of a finite group $G$. A long standing conjecture (ZC) attributed to $\mathrm{H}$. Zassenhaus says that every torsion unit $u \in V(\mathbb{Z} G)$ is conjugate within the rational group algebra $\mathbb{Q} G$ to an element in $G$ (see [25]).

For finite simple groups, the main tool for the investigation of the Zassenhaus conjecture is the Luthar-Passi method, introduced in [23] to solve it for $A_{5}$. Later in 20. M. Hertweck applied it to the investigation of (ZC) for $P S L\left(2, p^{n}\right)$ using Brauer character tables as well as ordinary ones. In [2, 7] the method of $(p, q)$-constant characters was introduced to optimize the Luthar-Passi method for elements of order $p q$. The approach of Luthar and Passi proved to be useful for groups containing non-trivial normal subgroups as well. For some recent results on (ZC) we refer to [3]-[11, [17, 19, 20, 22].

A weakened version of (ZC) can be stated using the notion of the GruenbergKegel graph (also called the prime graph) of an arbitrary group $X$, which is the graph $\pi(X)$ whose vertices are labeled by primes $p$ for which there exists an element of order $p$ in $X$ and with an edge from $p$ to a distinct $q$ if $X$ has an element of order $p q$. The following question is posted by W. Kimmerle in 24] (Problem 21):

(PQ) $\quad$ For a finite group $G$, is it true that $\pi(V(\mathbb{Z} G))=\pi(G)$ ?

Of course if (ZC) holds for a finite group $G$ then (PQ) has a positive answer for $G$. In 22] it is shown, in particular, that (PQ) has a positive answer for finite Frobenius

Received by the editor April 27, 2007 and, in revised form, September 7, 2009.

2010 Mathematics Subject Classification. Primary 16S34, 20C05; Secondary 20D08.

Key words and phrases. Zassenhaus conjecture, prime graph, torsion unit, partial augmentation, integral group ring.

The research was supported by OTKA No. K68383, Onderzoeksraad of Vrije Universiteit Brussel, Fonds voor Wetenschappelijk Onderzoek (Belgium), Flemish-Polish bilateral agreement BIL2005/VUB/2006, Francqui Stichting (Belgium) grant ADSI107 and The Royal Society of Edinburgh International Exchange Programme.

(c)2010 American Mathematical Society 
and solvable groups. For solvable groups this result was recently improved by M. Hertweck in [18, where it was shown that orders of torsion units in $V(\mathbb{Z} G)$ are exactly orders of $G$.

In 2, 4, 5, 6, 7, 8, 9, 11, the problem (PQ) was investigated for Mathieu, HigmanSims, McLaughlin, Held, Rudvalis, Suzuki and O'Nan sporadic simple groups. In this paper we continue these investigations for the Janko simple groups. For $J_{1}, J_{2}$ and $J_{3}$ we again give a positive answer to (PQ) and for $J_{4}$ the question remains open. In the final section we include some comments on the computational difficulties encountered for $J_{4}$.

Let $G$ be a group. Put $\mathcal{C}=\left\{C_{1}, \ldots, C_{n t}, \ldots\right\}$, the collection of all conjugacy classes of $G$, where the first index denotes the order of the elements of this conjugacy class and $C_{1}=\{1\}$ (throughout the paper we will use the ordering of conjugacy classes as used in the GAP Character Table Library). Suppose $u=\sum \alpha_{g} g \in V(\mathbb{Z} G)$ has finite order. Denote by $\nu_{n t}=\nu_{n t}(u)=\sum_{g \in C_{n t}} \alpha_{g}$, the partial augmentation of $u$ with respect to $C_{n t}$. From the Berman-Higman Theorem (see, for example, [1]) one knows that $\operatorname{tr}(u)=\nu_{1}=0$, and clearly

$$
\sum_{C_{n t} \in \mathcal{C}} \nu_{n t}=1
$$

Hence, for any character $\chi$ of $G$, we get that $\chi(u)=\sum \nu_{n t} \chi\left(h_{n t}\right)$, where $h_{n t}$ is a representative of a conjugacy class $C_{n t}$.

The main results for the Janko simple groups $J_{1}, J_{2}$ and $J_{3}$ are as follows.

Theorem 1. Let $G$ denote the first Janko simple group $J_{1}$. Let $u$ be a torsion unit of $V(\mathbb{Z} G)$ of order $|u|$ with the tuple of length 15 containing partial augmentations for all conjugacy classes of $G$. The following properties hold.

(i) There are no units of orders 14, 21, 22, 33, 35, 38, 55, 57, 77, 95, 133 and 209 in $V(\mathbb{Z} G)$. Equivalently, if $|u| \neq 30$, then $|u|$ coincides with the order of some element $g \in G$.

(ii) If $|u| \in\{2,3,7,11,19\}$, then $u$ is rationally conjugate to some $g \in G$.

(iii) If $|u|=5$, then $\nu_{k x}=0$ for $k x \notin\{5 a, 5 b\}$ and

$$
\left(\nu_{5 a}, \nu_{5 b}\right) \in\{(-1,2),(0,1),(1,0),(2,-1)\} .
$$

(iv) If $|u|=6$, then $\nu_{k x}=0$ for $k x \notin\{2 a, 3 a, 6 a\}$ and

$$
\begin{array}{r}
\left(\nu_{2 a}, \nu_{3 a}, \nu_{6 a}\right) \in\{(-4,3,2),(-2,0,3),(-2,3,0),(0,0,1), \\
(0,3,-2),(2,0,-1)\} .
\end{array}
$$

(v) If $|u|=10$, then $\nu_{k x}=0$ for $k x \notin\{5 a, 5 b, 10 a, 10 b\}$ and

$$
\begin{array}{r}
\left(\nu_{5 a}, \nu_{5 b}, \nu_{10 a}, \nu_{10 b}\right) \in\{(2,-2,0,1),(0,0,2,-1),(0,0,0,1),(-1,1,1,0), \\
(1,-1,-1,2),(1,-1,1,0),(-2,2,1,0),(0,0,-1,2),(0,0,1,0), \\
(-1,1,2,-1),(-1,1,0,1),(1,-1,0,1)\} .
\end{array}
$$

(vi) If $|u|=15$, then $\nu_{k x}=0$ for $k x \notin\{5 a, 5 b, 15 a, 15 b\}$ and then

$$
\left(\nu_{5 a}, \nu_{5 b}, \nu_{15 a}, \nu_{15 b}\right) \in\{(-1,1,0,1),(0,0,0,1),(0,0,1,0),(1,-1,1,0)\} .
$$


(vii) If $|u|=30$, then $\nu_{k x}=0$ for $k x \notin\{5 a, 5 b, 10 a, 10 b, 15 a, 15 b\}$ and

$$
\begin{array}{r}
\left(\nu_{5 a}, \nu_{5 b}, \nu_{10 a}, \nu_{10 b}, \nu_{15 a}, \nu_{15 b}\right) \in\{(-1,1,-1,-1,1,2), \\
(-1,1,-2,0,1,2),(0,0,-2,0,1,2),(0,0,0,-2,2,1), \\
(1,-1,-1,-1,2,1),(1,-1,0,-2,2,1)\} .
\end{array}
$$

Theorem 2. Let $G$ denote the second Janko simple group $J_{2}$. Let $u$ be a torsion unit of $V(\mathbb{Z} G)$ of order $|u|$ with the tuple of length 21 containing partial augmentations for all conjugacy classes of $G$. The following properties hold.

(i) There are no units of orders 14,21 and 35 in $V(\mathbb{Z} G)$. Equivalently, if $|u| \notin$ $\{20,24,30,40,60,120\}$, then $|u|$ coincides with the order of some $g \in G$.

(ii) If $|u| \in\{7,15\}$, then $u$ is rationally conjugate to some $g \in G$.

(iii) If $|u|=2$, then $\nu_{k x}=0$ for $k x \notin\{2 a, 2 b\}$ and

$$
\left(\nu_{2 a}, \nu_{2 b}\right) \in\{(0,1),(-2,3),(2,-1),(1,0),(3,-2),(-1,2)\} .
$$

(iv) If $|u|=3$, then $\nu_{k x}=0$ for $k x \notin\{3 a, 3 b\}$ and

$$
\left(\nu_{3 a}, \nu_{3 b}\right) \in\{(0,1),(1,0),(-1,2)\} .
$$

(v) If $|u|=4$, then $\nu_{k x}=0$ for $k x \notin\{2 a, 2 b, 4 a\}$ and

$$
\begin{array}{r}
\left(\nu_{2 a}, \nu_{2 b}, \nu_{4 a}\right) \in\{(-2,-2,5),(-1,-3,5),(-1,-1,3),(-1,1,1), \\
(0,-4,5),(0,-2,3),(0,0,1),(0,2,-1),(0,4,-3),(1,-3,3), \\
(1,-1,1),(1,1,-1),(1,3,-3),(2,0,-1),(2,2,-3)\} .
\end{array}
$$

(vi) If $|u|=5$, then $\nu_{k x}=0$ for $k x \notin\{5 a, 5 b, 5 c, 5 d\}$ and

$$
\begin{array}{r}
\left(\nu_{5 a}, \nu_{5 b}, \nu_{5 c}, \nu_{5 d}\right) \in\{(0,0,2,-1),(1,0,0,0),(0,0,0,1), \\
(0,0,-1,2),(1,0,-1,1),(0,0,1,0),(0,1,0,0), \\
(1,1,0,-1),(1,1,-1,0),(0,1,1,-1)\} .
\end{array}
$$

(vii) If $|u|=8$, then $\nu_{k x}=0$ for $k x \notin\{2 a, 2 b, 4 a, 8 a\}$ and

$$
\begin{gathered}
\left(\nu_{2 a}, \nu_{2 b}, \nu_{4 a}, \nu_{8 a}\right) \in\{(-1,-1,0,3),(-1,-1,2,1),(-1,1,-2,3), \\
(-1,1,0,1),(0,-2,0,3),(0,-2,2,1),(0,0,-2,3),(1,-1,2,-1), \\
(0,0,2,-1),(0,2,-2,1),(0,2,0,-1),(0,2,2,-3),(1,-1,0,1), \\
(0,0,0,1)(1,1,-2,1),(1,1,0,-1),(1,1,2,-3),(2,0,2,-3)\} .
\end{gathered}
$$

Theorem 3. Let $G$ denote the third Janko simple group $J_{3}$. Let $u$ be a torsion unit of $V(\mathbb{Z} G)$ of order $|u|$ with the tuple of length 21 containing partial augmentations for all conjugacy classes of $G$. The following properties hold.

(i) There are no units of orders $34,38,51,57,85,95$ and 323 in $V(\mathbb{Z} G)$. Equivalently, if $|u| \notin\{18,20,24,30,36,40,45,60,72,90,120,180,360\}$, then $|u|$ coincides with the order of some element $g \in G$.

(ii) If $|u|=2, u$ is rationally conjugate to some $g \in G$.

(iii) If $|u|=3$, then $\nu_{k x}=0$ for $k x \notin\{3 a, 3 b\}$ and

$$
\begin{array}{r}
\left(\nu_{3 a}, \nu_{3 b}\right) \in\{(5,-4),(0,1),(-2,3),(2,-1),(-3,4), \\
(-4,5),(1,0),(3,-2),(-1,2),(4,-3)\} .
\end{array}
$$

(iv) If $|u|=4$, then $\nu_{k x}=0$ for $k x \notin\{2 a, 4 a\}$ and

$$
\left(\nu_{2 a}, \nu_{4 a}\right) \in\{(0,1),(-2,3),(2,-1)\} .
$$


(v) If $|u|=5$, then $\nu_{k x}=0$ for $k x \notin\{5 a, 5 b\}$ and

$$
\begin{array}{r}
\left(\nu_{5 a}, \nu_{5 b}\right) \in\{(0,1),(-2,3),(2,-1),(-3,4),(1,0), \\
(3,-2),(-1,2),(4,-3)\} .
\end{array}
$$

(vi) If $|u|=8$, then $\nu_{k x}=0$ for $k x \notin\{2 a, 4 a, 8 a\}$ and

$$
\begin{array}{r}
\left(\nu_{2 a}, \nu_{4 a}, \nu_{8 a}\right) \in\{(-2,-6,9),(-2,-4,7),(-2,-2,5),(-2,0,3), \\
(-2,2,1),(0,-4,5),(0,-2,3),(0,0,1),(0,2,-1),(0,4,-3), \\
(2,-2,1),(2,0,-1),(2,2,-3),(2,4,-5),(2,6,-7)\} .
\end{array}
$$

(vii) If $|u|=17$, then $\nu_{k x}=0$ for $k x \notin\{17 a, 17 b\}$ and

$$
\begin{aligned}
\left(\nu_{17 a}, \nu_{17 b}\right) \in\{ & (5,-4),(0,1),(-2,3),(2,-1),(-3,4), \\
& (-4,5),(1,0),(3,-2),(-1,2),(4,-3)\} .
\end{aligned}
$$

(viii) If $|u|=19$, then $\nu_{k x}=0$ for $k x \notin\{19 a, 19 b\}$ and

$$
\begin{aligned}
\left(\nu_{19 a}, \nu_{19 b}\right) \in\{ & (5,-4),(0,1),(-2,3),(2,-1),(-3,4), \\
& (-4,5),(1,0),(3,-2),(-1,2),(4,-3)\} .
\end{aligned}
$$

As a consequence of the first parts of Theorems 1 3 we get the following.

Corollary 1. If $G \in\left\{J_{1}, J_{2}, J_{3}\right\}$, then $\pi(G)=\pi(V(\mathbb{Z} G))$.

\section{Preliminaries}

The following result relates the solution of the Zassenhaus conjecture to partial augmentations of torsion units.

Proposition 1 (see [23]). Let $u \in V(\mathbb{Z} G)$ be a torsion unit of order $k$. Then $u$ is conjugate in $\mathbb{Q} G$ to an element $g \in G$ if and only if for each $d$ dividing $k$ there is precisely one conjugacy class $C$ with partial augmentation $\varepsilon_{C}\left(u^{d}\right) \neq 0$.

The next result already yields that several partial augmentations are zero.

Proposition 2 (see [17, Proposition 3.1). Let $G$ be a finite group and let $u$ be a torsion unit in $V(\mathbb{Z} G)$. If $x$ is an element of $G$ whose p-part, for some prime $p$, has order strictly greater than the order of the p-part of $u$, then $\varepsilon_{x}(u)=0$.

The key restriction on partial augmentations is given by the following result.

Proposition 3 (see [20, 23]). Let either $p=0$ or $p$ be a prime divisor of $|G|$. Suppose that $u \in V(\mathbb{Z} G)$ has finite order $k$ and assume $k$ and $p$ are coprime in case $p \neq 0$. If $z$ is a complex primitive $k$-th root of unity and $\chi$ is either an ordinary character or a p-Brauer character of $G$, then, for every integer $l$, the number

$$
\mu_{l}(u, \chi, p)=\frac{1}{k} \sum_{d \mid k} \operatorname{Tr}_{\mathbb{Q}\left(z^{d}\right) / \mathbb{Q}}\left(\chi\left(u^{d}\right) z^{-d l}\right)
$$

is a non-negative integer.

Note that if $p=0$, we will use the notation $\mu_{l}(u, \chi, *)$ for $\mu_{l}(u, \chi, 0)$.

When $s$ and $t$ are two primes such that $G$ contains no element of order $s t$, and $u$ is a normalized torsion unit of order st, Proposition 3 may be reformulated as follows. Let $\nu_{k}$ be the sum of partial augmentations of $u$ with respect to all conjugacy classes of elements of order $k$ in $G$, i.e. $\nu_{2}=\nu_{2 a}+\nu_{2 b}$, etc. Then by (1) and Proposition 2 we obtain that $\nu_{s}+\nu_{t}=1$ and $\nu_{k}=0$ for $k \notin\{s, t\}$. For each character $\chi$ of 
$G$ (an ordinary character or a Brauer character in characteristic not dividing st) that is constant on all elements of orders $s$ and on all elements of order $t$, we have $\chi(u)=\nu_{s} \chi\left(C_{s}\right)+\nu_{t} \chi\left(C_{t}\right)$, where $\chi\left(C_{r}\right)$ denotes the value of the character $\chi$ on any element of order $r$ from $G$.

From Proposition 3 we obtain that the values

$$
\begin{aligned}
\mu_{l}(u, \chi, p)=\frac{1}{s t}(\chi(1) & +\operatorname{Tr}_{\mathbb{Q}\left(z^{s}\right) / \mathbb{Q}}\left(\chi\left(u^{s}\right) z^{-s l}\right) \\
& \left.+\operatorname{Tr}_{\mathbb{Q}\left(z^{t}\right) / \mathbb{Q}}\left(\chi\left(u^{t}\right) z^{-t l}\right)+\operatorname{Tr}_{\mathbb{Q}(z) / \mathbb{Q}}\left(\chi(u) z^{-l}\right)\right)
\end{aligned}
$$

are nonnegative integers. It follows that if $\chi$ has the specified property, then

$$
\mu_{l}(u, \chi, p)=\frac{1}{s t}\left(m_{1}+\nu_{s} m_{s}+\nu_{t} m_{t}\right)
$$

where

$$
\begin{aligned}
& m_{1}=\chi(1)+\chi\left(C_{t}\right) T r_{\mathbb{Q}\left(z^{s}\right) / \mathbb{Q}}\left(z^{-s l}\right)+\chi\left(C_{s}\right) \operatorname{Tr} r_{\mathbb{Q}\left(z^{t}\right) / \mathbb{Q}}\left(z^{-t l}\right), \\
& m_{s}=\chi\left(C_{s}\right) T r_{\mathbb{Q}(z) / \mathbb{Q}}\left(z^{-l}\right), \quad m_{t}=\chi\left(C_{t}\right) \operatorname{Tr}_{\mathbb{Q}(z) / \mathbb{Q}}\left(z^{-l}\right) .
\end{aligned}
$$

Finally, we shall use well-known restrictions for torsion units.

Proposition 4 (12]). The order of a torsion element $u \in V(\mathbb{Z} G)$ divides $\exp (G)$.

Proposition 5 (see [12]). Let $p$ be a prime, and let $u$ be a torsion unit of $V(\mathbb{Z} G)$ of order $p^{n}$. Then for $m \neq n$ the sum of all partial augmentations of $u$ with respect to conjugacy classes of elements of order $p^{m}$ is divisible by $p$.

\section{Proof of Theorem 1}

In this section we denote by $G$ the first Janko simple group $J_{1}$. It is well known 13, 15] that $|G|=2^{3} \cdot 3 \cdot 5 \cdot 7 \cdot 11 \cdot 19$ and $\exp (G)=2 \cdot 3 \cdot 5 \cdot 7 \cdot 11 \cdot 19$. The character table of $G$, as well as the Brauer character tables for $p \in\{2,3,5,7,11,19\}$, denoted by $\mathfrak{B C T}(p)$, can be found using the computational algebra system GAP [15, which derives its data from [13, 21. We will use the notation, including the indices, for the characters and conjugacy classes as used in the GAP Character Table Library.

Since $G$ only possesses elements of orders $2,3,5,6,7,10,11,15$ and 19, we first investigate normalized units of these orders. After this, by Proposition 4 , the order of each torsion unit divides $\exp (G)$, so it is enough to consider normalized units of orders $14,21,22,30,33,35,38,55,57,77,95,133$ and 209 , because if $u$ is a unit of another possible order, then there is $t \in \mathbb{N}$ such that $u^{t}$ has an order from this list. We shall prove that units of all these orders except 30 do not appear in $V(\mathbb{Z} G)$.

Assume that $u$ is a non-trivial normalized unit and consider each case separately. 
- Let $|u| \in\{2,3,7,11\}$. Since there is only one conjugacy class in $G$ consisting of elements of order $|u|$, this case follows at once from Propositions 1 and 2 .

- Let $|u|=19$. By (1) and Proposition 2 we have that $\nu_{19 a}+\nu_{19 b}+\nu_{19 c}=1$. Applying Proposition 3 to characters $\chi_{2}, \chi_{8}, \chi_{13}$ in $\mathfrak{B C T}(11)$ we get the system:

$$
\begin{array}{cl}
\mu_{1}\left(u, \chi_{2}, 11\right)=\frac{1}{19}\left(-t_{1}+7\right) \geq 0 ; & \mu_{1}\left(u, \chi_{8}, 11\right)=\frac{1}{19}\left(t_{1}+69\right) \geq 0 \\
\mu_{2}\left(u, \chi_{2}, 11\right)=\frac{1}{19}\left(-t_{2}+7\right) \geq 0 ; & \mu_{2}\left(u, \chi_{8}, 11\right)=\frac{1}{19}\left(t_{2}+69\right) \geq 0 \\
\mu_{4}\left(u, \chi_{2}, 11\right)=\frac{1}{19}\left(t_{3}+7\right) \geq 0 ; \quad \mu_{4}\left(u, \chi_{8}, 11\right)=\frac{1}{19}\left(-t_{3}+69\right) \geq 0 \\
\mu_{1}\left(u, \chi_{13}, 11\right)=\frac{1}{19}\left(14 \nu_{19 a}-5 \nu_{19 b}-5 \nu_{19 c}+119\right) \geq 0 ; \\
\mu_{2}\left(u, \chi_{13}, 11\right)=\frac{1}{19}\left(-5 \nu_{19 a}+14 \nu_{19 b}-5 \nu_{19 c}+119\right) \geq 0 \\
\mu_{4}\left(u, \chi_{13}, 11\right)=\frac{1}{19}\left(-5 \nu_{19 a}-5 \nu_{19 b}+14 \nu_{19 c}+119\right) \geq 0 .
\end{array}
$$

where $t_{1}=7 \nu_{19 a}-12 \nu_{19 b}+7 \nu_{19 c}, t_{2}=7 \nu_{19 a}+7 \nu_{19 b}-12 \nu_{19 c}$ and $t_{3}=12 \nu_{19 a}-$ $7 \nu_{19 b}-7 \nu_{19 c}$. From these restrictions and the requirement that all $\mu_{i}\left(u, \chi_{j}, p\right)$ must be non-negative integers we get that $\left(\nu_{19 a}, \nu_{19 b}, \nu_{19 c}\right) \in\{(1,0,0),(0,1,0),(0,0,1)\}$.

Thus, for units of orders $2,3,7,11$ and 19 there is precisely one conjugacy class with non-zero partial augmentation, so Proposition 1 yields part (ii) of Theorem 1 .

Note that using the LAGUNA package [10] in combination with constraint solvers MINION [16] and ECLiPSe [14, we computed inequalities from Proposition 3 for every irreducible character from ordinary and Brauer character tables, and for every $0 \leq l \leq|u|-1$ (it is enough to enumerate $l$ in this range since $z^{|u|}=1$, so for bigger values of $l$ we will not have new inequalities), but the only inequalities that really matter are those listed above. The same remark applies for all other orders of torsion units considered in this paper.

- Let $u$ be a unit of order 5. By (1) and Proposition 2 we get $\nu_{5 a}+\nu_{5 b}=1$. Again applying Proposition 3 to characters in $\mathfrak{B C T}(11)$ we get the following system of inequalities:

$$
\begin{array}{ll}
\mu_{1}\left(u, \chi_{2}, 11\right)=\frac{1}{5}\left(3 \nu_{5 a}-2 \nu_{5 b}+7\right) \geq 0 ; & \mu_{2}\left(u, \chi_{2}, 11\right)=\frac{1}{5}\left(-2 \nu_{5 a}+3 \nu_{5 b}+7\right) \geq 0 ; \\
\mu_{1}\left(u, \chi_{3}, 11\right)=\frac{1}{5}\left(-4 \nu_{5 a}+\nu_{5 b}+14\right) \geq 0 ; & \mu_{2}\left(u, \chi_{3}, 11\right)=\frac{1}{5}\left(\nu_{5 a}-4 \nu_{5 b}+14\right) \geq 0 ; \\
\mu_{1}\left(u, \chi_{5}, 11\right)=\frac{1}{5}\left(\nu_{5 a}-4 \nu_{5 b}+49\right) \geq 0 ; & \mu_{1}\left(u, \chi_{6}, 11\right)=\frac{1}{5}\left(-6 \nu_{5 a}+4 \nu_{5 b}+56\right) \geq 0 ; \\
\mu_{2}\left(u, \chi_{6}, 11\right)=\frac{1}{5}\left(4 \nu_{5 a}-6 \nu_{5 b}+56\right) \geq 0 ; & \mu_{2}\left(u, \chi_{7}, 11\right)=\frac{1}{5}\left(-4 \nu_{5 a}+6 \nu_{5 b}+64\right) \geq 0 ; \\
\mu_{2}\left(u, \chi_{8}, 11\right)=\frac{1}{5}\left(\nu_{5 a}-4 \nu_{5 b}+69\right) \geq 0 ; & \mu_{1}\left(u, \chi_{12}, 11\right)=\frac{1}{5}\left(4 \nu_{5 a}-\nu_{5 b}+106\right) \geq 0,
\end{array}
$$

that has only four integer solutions $\left(\nu_{5 a}, \nu_{5 b}\right) \in\{(0,1),(2,-1),(1,0),(-1,2)\}$ such that all $\mu_{i}\left(u, \chi_{j}, 11\right)$ are non-negative integers, so part (iii) of Theorem 1 is proved.

- Let $|u|=6$. By (1) and Proposition 2 we have that $\nu_{2 a}+\nu_{3 a}+\nu_{6 a}=1$. Applying Proposition 3 to characters in $\mathfrak{B C T}(11)$ we get the following system of inequalities:

$$
\begin{array}{ll}
\mu_{3}\left(u, \chi_{4}, 11\right)=\frac{1}{6}\left(-6 \nu_{2 a}+24\right) \geq 0 ; & \mu_{0}\left(u, \chi_{4}, 11\right)=\frac{1}{6}\left(6 \nu_{2 a}+30\right) \geq 0 ; \\
\mu_{0}\left(u, \chi_{6}, 11\right)=\frac{1}{6}\left(4 \nu_{3 a}+60\right) \geq 0 ; & \mu_{3}\left(u, \chi_{6}, 11\right)=\frac{1}{6}\left(-4 \nu_{3 a}+60\right) \geq 0 ; \\
\mu_{0}\left(u, \chi_{2}, 11\right)=\frac{1}{6}\left(-t_{1}+8\right) \geq 0 ; & \mu_{3}\left(u, \chi_{2}, 11\right)=\frac{1}{6}\left(t_{1}+10\right) \geq 0 ; \\
\mu_{0}\left(u, \chi_{3}, 11\right)=\frac{1}{6}\left(-2 t_{2}+10\right) \geq 0 ; & \mu_{1}\left(u, \chi_{3}, 11\right)=\frac{1}{6}\left(-t_{2}+17\right) \geq 0 ; \\
\mu_{3}\left(u, \chi_{3}, 11\right)=\frac{1}{6}\left(2 t_{2}+14\right) \geq 0,
\end{array}
$$


where $t_{1}=2 \nu_{2 a}-2 \nu_{3 a}+2 \nu_{6 a}$ and $t_{2}=2 \nu_{2 a}+\nu_{3 a}-\nu_{6 a}$ which has only six integer solutions such that all $\mu_{i}\left(u, \chi_{j}, 11\right)$ are non-negative integers. These are as listed in part (iv) of Theorem 1

- Let $|u|=10$. By (11) and Proposition 2 we get $\nu_{2 a}+\nu_{5 a}+\nu_{5 b}+\nu_{10 a}+\nu_{10 b}=1$. For any character $\chi$ of $G$ we need to consider 4 cases from part (iii) of Theorem 1 ,

Case 1. $\chi\left(u^{2}\right)=\chi(5 a)$.

Case 2. $\chi\left(u^{2}\right)=\chi(5 b)$.

Case 3. $\chi\left(u^{2}\right)=2 \chi(5 a)-\chi(5 b)$.

Case 4. $\chi\left(u^{2}\right)=-\chi(5 a)+2 \chi(5 b)$.

Here and below, $\chi(5 a)$ denotes the value of the character $\chi$ on the representative of the conjugacy class $C_{5 a}$, etc.

Applying Proposition 3 to characters in $\mathfrak{B C T}(11)$ we get the system:

$$
\begin{array}{ll}
\mu_{0}\left(u, \chi_{2}, 11\right)=\frac{1}{10}\left(-t_{1}+4\right) \geq 0 ; & \mu_{5}\left(u, \chi_{2}, 11\right)=\frac{1}{10}\left(t_{1}+6\right) \geq 0 ; \\
\mu_{2}\left(u, \chi_{3}, 11\right)=\frac{1}{10}\left(t_{2}+\alpha_{1}\right) \geq 0 ; & \mu_{3}\left(u, \chi_{3}, 11\right)=\frac{1}{10}\left(-t_{2}+\alpha_{2}\right) \geq 0 ; \\
\mu_{0}\left(u, \chi_{6}, 11\right)=\frac{1}{10}\left(t_{3}+60\right) \geq 0 ; & \mu_{5}\left(u, \chi_{6}, 11\right)=\frac{1}{10}\left(-t_{3}+60\right) \geq 0 ; \\
\mu_{1}\left(u, \chi_{6}, 11\right)=\frac{1}{10}\left(-t_{4}+\alpha_{3}\right) \geq 0 ; & \mu_{4}\left(u, \chi_{6}, 11\right)=\frac{1}{10}\left(t_{4}+\alpha_{3}\right) \geq 0,
\end{array}
$$

where $t_{1}=4 \nu_{2 a}+2 \nu_{5 a}+2 \nu_{5 b}-6 \nu_{10 a}-6 \nu_{10 b}, \quad t_{2}=2 \nu_{2 a}-4 \nu_{5 a}+\nu_{5 b}+2 \nu_{10 a}-3 \nu_{10 b}$, $t_{3}=4 \nu_{5 a}+4 \nu_{5 b}$ and $t_{4}=4 \nu_{5 a}-6 \nu_{5 b}$, and $\left(\alpha_{1}, \alpha_{2}, \alpha_{3}\right)$ is equal to $(13,17,50)$, $(8,12,60),(18,22,40)$ and $(3,7,70)$ in cases $1-4$ respectively.

Now denote $t_{1}=2 \nu_{2 a}+\nu_{5 a}+\nu_{5 b}-3 \nu_{10 a}-3 \nu_{10 b} \in \mathbb{Z}$, then from the first two inequalities (recall that all $\mu_{i}\left(u, \chi_{j}, p\right)$ must be non-negative integers) we obtain that $t_{1} \in\{-3,2\}$. Put $t_{2}=2 \nu_{2 a}-4 \nu_{5 a}+\nu_{5 b}+2 \nu_{10 a}-3 \nu_{10 b} \in \mathbb{Z}$. From the third and fourth inequalities $t_{2}$ belongs to the set $\{-13,-3,7,17\},\{-8,2,12\}$, $\{-18,-8,2,12,22\}$ and $\{-3,7\}$ in cases $1-4$ respectively. Put $t_{3}=\nu_{5 a}+\nu_{5 b} \in \mathbb{Z}$. From the fifth and eighth inequalities it follows that $t_{3} \in\{5 k \mid-3 \leq k \leq 3\}$.

Finally, put $t_{4}=2 \nu_{5 a}-3 \nu_{5 b}$. Considering the sixth and seventh inequalities we get that $t_{4}$ belongs to the set $\{5 k \mid-5 \leq k \leq 5\},\{5 k \mid-6 \leq k \leq 6\}$, $\{5 k \mid-4 \leq k \leq 4\}$ and $\{5 k \mid-7 \leq k \leq 7\}$ in cases $1-4$ respectively.

We obtain the system of five linear equations:

$$
\begin{aligned}
\nu_{2 a}+\nu_{5 a}+\nu_{5 b}+\nu_{10 a}+\nu_{10 b} & =1 ; \\
2 \nu_{2 a}+\nu_{5 a}+\nu_{5 b}-3 \nu_{10 a}-3 \nu_{10 b} & =t_{1} ; \\
2 \nu_{2 a}-4 \nu_{5 a}+\nu_{5 b}+2 \nu_{10 a}-3 \nu_{10 b} & =t_{2} ; \\
\nu_{5 a}+\nu_{5 b}=t_{3} ; \quad 2 \nu_{5 a}-3 \nu_{5 b} & =t_{4} .
\end{aligned}
$$

Since the matrix of the system is non-degenerate, it has a unique solution for any values of parameters $t_{i}$. For each of the allowable values of $t_{1}, t_{2}, t_{3}$ and $t_{4}$ we can thus compute the unique integer solution of this system of equations.

Now Proposition 3 for $\mathfrak{B C T}(11)$ also gives the following additional inequalities:

$$
\begin{aligned}
& \mu_{2}\left(u, \chi_{2}, 11\right)=\frac{1}{10}\left(t_{1}+\beta_{2}\right) \geq 0 ; \quad \mu_{3}\left(u, \chi_{2}, 11\right)=\frac{1}{10}\left(-t_{1}+\beta_{3}\right) \geq 0 ; \\
& \mu_{1}\left(u, \chi_{2}, 11\right)=\frac{1}{10}\left(-t_{2}+\beta_{1}\right) \geq 0 ; \quad \mu_{4}\left(u, \chi_{2}, 11\right)=\frac{1}{10}\left(t_{2}+\beta_{4}\right) \geq 0 ; \\
& \mu_{1}\left(u, \chi_{3}, 11\right)=\frac{1}{10}\left(-t_{3}+\beta_{5}\right) \geq 0 ; \quad \mu_{4}\left(u, \chi_{3}, 11\right)=\frac{1}{10}\left(t_{3}+\beta_{6}\right) \geq 0, \\
& \mu_{0}\left(u, \chi_{3}, 11\right)=\frac{1}{10}\left(-8 \nu_{2 a}+6 \nu_{5 a}+6 \nu_{5 b}+2 \nu_{10 a}+2 \nu_{10 b}+18\right) \geq 0 ;
\end{aligned}
$$


$t_{1}=\nu_{2 a}+3 \nu_{5 a}-2 \nu_{5 b}+\nu_{10 a}-4 \nu_{10 b}, t_{2}=\nu_{2 a}-2 \nu_{5 a}+3 \nu_{5 b}-4 \nu_{10 a}+\nu_{10 b}$ and $t_{3}=2 \nu_{2 a}+\nu_{5 a}-4 \nu_{5 b}-3 \nu_{10 a}+2 \nu_{10 b}$, and $\left(\beta_{1}, \beta_{2}, \beta_{3}, \beta_{4}, \beta_{5}, \beta_{6}\right)$ is equal to $(11,-4,6,9,12,8),(6,9,11,4,17,13),(16,-1,1,14,7,3),(1,14,16,-1,22,18)$ in cases 1-4 respectively. It then follows that $\nu_{2 a}=0$ and the only integer solutions with non-negative integers $\mu_{i}\left(u, \chi_{j}, 11\right)$ are those listed in part (v) of Theorem 1 .

- Let $|u|=15$. By (1) and Proposition 2 we get $\nu_{3 a}+\nu_{5 a}+\nu_{5 b}+\nu_{15 a}+\nu_{15 b}=1$. Since $\left|u^{3}\right|=5$, for any character $\chi$ of $G$ we need to consider four cases, defined by part (iii) of Theorem 1 ;

Case 1. $\chi\left(u^{3}\right)=\chi(5 a)$.

Case 2. $\chi\left(u^{3}\right)=\chi(5 b)$.

Case 3. $\chi\left(u^{3}\right)=2 \chi(5 a)-\chi(5 b)$.

Case 4. $\chi\left(u^{3}\right)=-\chi(5 a)+2 \chi(5 b)$.

Again applying Proposition 3 to characters in $\mathfrak{B C T}(11)$ we get the system:

$$
\begin{aligned}
& \mu_{0}\left(u, \chi_{2}, 11\right)=\frac{1}{15}\left(-2 t_{1}+7\right) \geq 0 ; \quad \mu_{5}\left(u, \chi_{2}, 11\right)=\frac{1}{15}\left(t_{1}+4\right) \geq 0 ; \\
& \mu_{0}\left(u, \chi_{3}, 11\right)=\frac{1}{15}\left(-2 t_{2}+18\right) \geq 0 ; \quad \mu_{5}\left(u, \chi_{3}, 11\right)=\frac{1}{15}\left(t_{2}+21\right) \geq 0 ; \\
& \mu_{1}\left(u, \chi_{4}, 11\right)=\frac{1}{15}\left(t_{3}+\alpha_{1}\right) \geq 0 ; \quad \mu_{6}\left(u, \chi_{4}, 11\right)=\frac{1}{15}\left(-2 t_{3}+\alpha_{1}\right) \geq 0 ; \\
& \mu_{1}\left(u, \chi_{6}, 11\right)=\frac{1}{15}\left(t_{4}+\alpha_{2}\right) \geq 0 ; \quad \mu_{1}\left(u, \chi_{7}, 11\right)=\frac{1}{15}\left(-t_{4}+\alpha_{3}\right) \geq 0,
\end{aligned}
$$

where $t_{1}=-4 \nu_{3 a}+2 \nu_{5 a}+2 \nu_{5 b}-4 \nu_{15 a}-4 \nu_{15 b}, t_{2}=4 \nu_{3 a}-6 \nu_{5 a}-6 \nu_{5 b}-6 \nu_{15 a}-6 \nu_{15 b}$, $t_{3}=-3 \nu_{5 a}+2 \nu_{5 b}+5 \nu_{15 b}$ and $t_{4}=2 \nu_{3 a}-4 \nu_{5 a}+6 \nu_{5 b}+2 \nu_{15 a}-3 \nu_{15 b}$, and $\left(\alpha_{1}, \alpha_{2}, \alpha_{3}\right)$ is equal to $(25,48,72),(30,58,62),(20,38,82)$ and $(35,68,52)$ in cases $1-4$ respectively.

Now denote $t_{1}=2 \nu_{3 a}-\nu_{5 a}-\nu_{5 b}+2 \nu_{15 a}+2 \nu_{15 b} \in \mathbb{Z}$, then from the first two inequalities (again recall that all $\mu_{i}\left(u, \chi_{j}, p\right)$ must be non-negative integers) we obtain that $t_{1}=2$. Put $t_{2}=2 \nu_{3 a}-3 \nu_{5 a}-3 \nu_{5 b}-3 \nu_{15 a}-3 \nu_{15 b} \in \mathbb{Z}$. From the third and fourth inequalities $t_{2}=-3$. Put $t_{3}=3 \nu_{5 a}-2 \nu_{5 b}-5 \nu_{15 b} \in \mathbb{Z}$. From the fifth and sixth inequalities it follows that $t_{3}$ belongs to the set $\{-5,10,25\}$, $\{-15,0,15,30\},\{-10,5,20\}$ and $\{-10,5,20,35\}$ in cases $1-4$ respectively.

Finally, put $t_{4}=2 \nu_{3 a}-4 \nu_{5 a}+6 \nu_{5 b}+2 \nu_{15 a}-3 \nu_{15 b} \in \mathbb{Z}$. Considering the seventh and eighth inequalities we get that $t_{4}$ belongs to the set $\{\gamma+15 k \mid k=0, \ldots, 8\}$, where $\gamma$ is equal to $-48,-58,-38,-68$ in cases $1-4$ respectively. So we get

$$
\begin{aligned}
\nu_{3 a}+\nu_{5 a}+\nu_{5 b}+\nu_{15 a}+\nu_{15 b} & =1 ; \\
2 \nu_{3 a}-\nu_{5 a}-\nu_{5 b}+2 \nu_{15 a}+2 \nu_{15 b} & =2 ; \\
2 \nu_{3 a}-3 \nu_{5 a}-3 \nu_{5 b}-3 \nu_{15 a}-3 \nu_{15 b} & =-3 ; \\
3 \nu_{5 a}-2 \nu_{5 b}-5 \nu_{15 b} & =t_{3} ; \\
2 \nu_{3 a}-4 \nu_{5 a}+6 \nu_{5 b}+2 \nu_{15 a}-3 \nu_{15 b} & =t_{4} .
\end{aligned}
$$

Since the matrix of the system is non-degenerate, again such a system has a unique solution for any values of the parameters $t_{i}$. For each of the allowable values of $t_{3}$ and $t_{4}$, we thus can compute the unique integer solution of this system of equations.

Now Proposition 3 for $\mathfrak{B C T}(11)$ also gives the following additional inequalities:

$$
\begin{array}{ll}
\mu_{2}\left(u, \chi_{2}, 11\right)=\frac{1}{15}\left(t_{1}+\beta_{2}\right) \geq 0 ; & \mu_{3}\left(u, \chi_{2}, 11\right)=\frac{1}{15}\left(-t_{1}+\beta_{3}\right) \geq 0 ; \\
\mu_{1}\left(u, \chi_{2}, 11\right)=\frac{1}{15}\left(t_{2}+\beta_{1}\right) \geq 0 ; & \mu_{6}\left(u, \chi_{2}, 11\right)=\frac{1}{15}\left(-2 t_{2}+\beta_{4}\right) \geq 0 ; \\
\mu_{1}\left(u, \chi_{3}, 11\right)=\frac{1}{15}\left(t_{3}+\beta_{5}\right) \geq 0 ; & \mu_{6}\left(u, \chi_{3}, 11\right)=\frac{1}{15}\left(-2 t_{3}+\beta_{7}\right) \geq 0 ; \\
\mu_{3}\left(u, \chi_{3}, 11\right)=\frac{1}{15}\left(2 \nu_{3 a}-8 \nu_{5 a}+2 \nu_{5 b}-8 \nu_{15 a}+2 \nu_{15 b}+\beta_{6}\right) \geq 0,
\end{array}
$$


where $t_{1}=\nu_{3 a}-3 \nu_{5 a}+2 \nu_{5 b}+6 \nu_{15 a}-4 \nu_{15 b}, t_{2}=\nu_{3 a}+2 \nu_{5 a}-3 \nu_{5 b}-4 \nu_{15 a}+6 \nu_{15 b}$ and $t_{3}=-\nu_{3 a}-\nu_{5 a}+4 \nu_{5 b}-\nu_{15 a}+4 \nu_{15 b}$, and $\left(\beta_{1}, \beta_{2}, \beta_{3}, \beta_{4}, \beta_{5}, \beta_{6}, \beta_{7}\right)$ is equal to $(9,4,7,12,11,13,8),(4,9,12,7,16,8,13),(14,-1,2,17,6,18,3)$ and $(-1,14,17,2$, $21,3,18)$ in cases $1-4$ respectively. It then follows that $\nu_{3 a}=0$ and the only integer solutions with non-negative integers $\mu_{i}\left(u, \chi_{j}, 11\right)$ are those listed in part (vi) of Theorem 1 .

- Let $|u|=30$. Since $\left|u^{i}\right|=\frac{|u|}{(|u|, i)}$, by parts (iii)-(vi) of Theorem 1 there are 4, 6, 12 and 4 tuples of partial augmentations for orders 5, 6, 10, and 15 respectively, so we need to consider cases $4 \cdot 6 \cdot 12 \cdot 4=1152$. Using the LAGUNA package [10] together with MINION and ECLiPSe [14, 16], we constructed and solved all of them, and only six cases given in the table below yield a non-trivial solution (see part (vii) of Theorem 1).

\begin{tabular}{|c|c|c|c|}
\hline$\chi\left(u^{6}\right)$ & $\chi\left(u^{5}\right)$ & $\chi\left(u^{3}\right)$ & $\chi\left(u^{2}\right)$ \\
\hline$\chi(5 b)$ & $3 \chi(3 a)-2 \chi(2 a)$ & $2 \chi(5 b)-2 \chi(5 a)+\chi(10 a)$ & $\chi(15 a)$ \\
$\chi(5 b)$ & $3 \chi(3 a)-2 \chi(2 a)$ & $2 \chi(10 b)-\chi(10 a)$ & $\chi(15 a)$ \\
$\chi(5 a)$ & $3 \chi(3 a)-2 \chi(2 a)$ & $2 \chi(10 a)-\chi(10 b)$ & $\chi(15 b)$ \\
$\chi(5 a)$ & $3 \chi(3 a)-2 \chi(2 a)$ & $2 \chi(5 a)-2 \chi(5 b)+\chi(10 b)$ & $\chi(15 b)$ \\
$2 \chi(5 a)-\chi(5 b)$ & $3 \chi(3 a)-2 \chi(2 a)$ & $\chi(10 a)$ & $\chi(5 a)-\chi(5 b)+\chi(15 a)$ \\
$2 \chi(5 b)-\chi(5 a)$ & $3 \chi(3 a)-2 \chi(2 a)$ & $\chi(10 b)$ & $\chi(5 b)-\chi(5 a)+\chi(15 b)$ \\
\hline
\end{tabular}

- It remains to prove part (i) of Theorem 1, that is, to show that $V(\mathbb{Z} G)$ has no elements of orders $14,21,22,33,35,38,55,57,77,95,133$ and 209 . We give a detailed proof for order 33. Other cases can be derived similarly from the table below containing the data for the constraints on partial augmentations $\nu_{p}$ and $\nu_{q}$ for possible orders $p q$ (including order 33 as well) accordingly to (2)-(4).

If $|u|=33$, then $\nu_{3}+\nu_{11}=1$. Consider ordinary characters $\xi=\chi_{7}$ and $\tau=\chi_{6}$, which are encoded in the table as $\xi=(7)_{[*]}$ and $\tau=(6)_{[*]}$ respectively. These characters are constant on elements of order 3 and elements of order 11: $\xi\left(C_{3}\right)=2$, $\xi\left(C_{11}\right)=0, \tau\left(C_{3}\right)=-1$ and $\tau\left(C_{11}\right)=0$. Now we obtain the following system:

$$
\begin{gathered}
\mu_{0}(u, \xi, *)=\frac{1}{33}\left(40 \nu_{3}+81\right) \geq 0 ; \quad \mu_{1}(u, \xi, *)=\frac{1}{33}\left(2 \nu_{3}+75\right) \geq 0 ; \\
\mu_{0}(u, \tau, *)=\frac{1}{33}\left(-20 \nu_{3}+75\right) \geq 0
\end{gathered}
$$

which has no integral solution $\left(\nu_{3}, \nu_{11}\right)$ such that all $\mu_{i}\left(u, \chi_{j}, *\right)$ are non-negative integers.

To complete the proof, we give the data for part (i) of Theorem 1 in the table below (we use the notation $\xi=(i)_{[p]}$ for $p$-Brauer characters). 


\begin{tabular}{|c|c|c|c|c|c|c|c|c|c|}
\hline$|u|$ & $p$ & $q$ & $\xi, \tau$ & $\xi\left(C_{p}\right)$ & $\xi\left(C_{q}\right)$ & $l$ & $m_{1}$ & $m_{p}$ & $m_{q}$ \\
\hline \multirow{3}{*}{14} & \multirow{3}{*}{2} & \multirow{3}{*}{7} & \multirow{3}{*}{$\xi=(6)_{[*]}$} & \multirow{3}{*}{5} & \multirow{3}{*}{0} & 0 & 82 & 30 & 0 \\
\hline & & & & & & 1 & 72 & 5 & 0 \\
\hline & & & & & & 7 & 72 & -30 & 0 \\
\hline \multirow{3}{*}{21} & \multirow{3}{*}{3} & \multirow{3}{*}{7} & \multirow{3}{*}{$\xi=(2)_{[*]}$} & \multirow{3}{*}{2} & \multirow{3}{*}{0} & 0 & 60 & 24 & 0 \\
\hline & & & & & & 1 & 54 & 2 & 0 \\
\hline & & & & & & 7 & 54 & -12 & 0 \\
\hline \multirow{3}{*}{22} & \multirow{3}{*}{2} & \multirow{3}{*}{11} & \multirow{3}{*}{$\xi=(6)_{[*]}$} & \multirow{3}{*}{5} & \multirow{3}{*}{0} & 0 & 82 & 50 & 0 \\
\hline & & & & & & 1 & 72 & 5 & 0 \\
\hline & & & & & & 7 & 72 & -50 & 0 \\
\hline \multirow{3}{*}{33} & \multirow{3}{*}{3} & \multirow{3}{*}{11} & $\xi=(7)_{[*]}$ & 2 & 0 & 0 & 81 & 40 & 0 \\
\hline & & & $\xi=(7)_{[*]}$ & 2 & 0 & 1 & 75 & 2 & 0 \\
\hline & & & $\tau=(6)_{[*]}$ & -1 & 0 & 0 & 75 & -20 & 0 \\
\hline \multirow{3}{*}{35} & \multirow{3}{*}{5} & \multirow{3}{*}{7} & $\xi=(6)_{[*]}$ & 2 & 0 & 0 & 85 & 48 & 0 \\
\hline & & & $\xi=(6)_{[*]}$ & 2 & 0 & 1 & 75 & 2 & 0 \\
\hline & & & $\tau=(12)_{[*]}$ & -2 & 0 & 0 & 125 & -48 & 0 \\
\hline \multirow{3}{*}{38} & \multirow{3}{*}{2} & & $\xi=(15)_{[*]}$ & 1 & 0 & 1 & 208 & 1 & 0 \\
\hline & & 19 & $\tau=(4)_{[*]}$ & 4 & 0 & 0 & 80 & 72 & 0 \\
\hline & & & $\tau=(4)_{[*]}$ & 4 & 0 & 19 & 72 & -72 & 0 \\
\hline & & & $\xi=(6)_{[*]}$ & 2 & 0 & 11 & 75 & -20 & 0 \\
\hline 55 & 5 & 11 & $\tau=(9)_{[*]}$ & 0 & -1 & 0 & 110 & 0 & -40 \\
\hline & & & $\tau=(9)_{[*]}$ & 0 & -1 & 5 & 121 & 0 & 4 \\
\hline & & & & & & 0 & 72 & -72 & 0 \\
\hline 57 & 3 & 19 & $\xi=(7)_{[2]}$ & -2 & 0 & 1 & 78 & -2 & 0 \\
\hline & & & & & & 19 & 78 & 36 & 0 \\
\hline & & & & & & 0 & 66 & 0 & 60 \\
\hline 77 & 7 & 11 & $\xi=(3)_{[2]}$ & 0 & 1 & 7 & 55 & 0 & -6 \\
\hline & & & & & & 11 & 66 & 0 & -10 \\
\hline & & & & & & 0 & 38 & 0 & 72 \\
\hline 95 & 5 & 19 & $\xi=(2)_{[2]}$ & 0 & 1 & 5 & 19 & 0 & -4 \\
\hline & & & & & & 19 & 38 & 0 & -18 \\
\hline & & & & & & 0 & 38 & 0 & -108 \\
\hline 133 & 7 & 19 & $\xi=(3)_{[2]}$ & 0 & -1 & 7 & 57 & 0 & 6 \\
\hline & & & & & & 19 & 38 & 0 & 18 \\
\hline & & & & & & 0 & 66 & -180 & 0 \\
\hline 209 & 11 & 19 & $\xi=(7)_{[2]}$ & -1 & 0 & 11 & 66 & 10 & 0 \\
\hline & & & & & & 19 & 77 & 18 & 0 \\
\hline
\end{tabular}

\section{Proof of Theorem 2}

Let $G$ be the second Janko simple group $J_{2}$. It is well known [13, 15, that $|G|=2^{7} \cdot 3^{3} \cdot 5^{2} \cdot 7$ and $\exp (G)=2^{3} \cdot 3 \cdot 5 \cdot 7$.

Since the group $G$ only possesses elements of orders $2,3,4,5,6,7,8,10,12$ and 15 , we will first investigate normalized units of these orders. Due to Proposition 4 . the order of each torsion unit divides the exponent of $G$, so it remains to consider normalized units of orders $14,20,21,24,30$ and 35 . We shall prove that units of all these orders except 20, 24 and 30 do not appear in $V(\mathbb{Z} G)$.

Assume that $u$ is a non-trivial normalized unit and consider each case separately. 
- Let $|u|=2$. By (11) and Proposition 2 we get $\nu_{2 a}+\nu_{2 b}=1$. By Proposition 3 we get the system of inequalities:

$\mu_{0}\left(u, \chi_{2}, *\right)=\frac{1}{2}\left(-2 \nu_{2 a}+2 \nu_{2 b}+14\right) \geq 0 ; \quad \mu_{1}\left(u, \chi_{2}, *\right)=\frac{1}{2}\left(2 \nu_{2 a}-2 \nu_{2 b}+14\right) \geq 0 ;$ $\mu_{0}\left(u, \chi_{4}, *\right)=\frac{1}{2}\left(5 \nu_{2 a}-3 \nu_{2 b}+21\right) \geq 0 ; \quad \mu_{1}\left(u, \chi_{4}, *\right)=\frac{1}{2}\left(-5 \nu_{2 a}+3 \nu_{2 b}+21\right) \geq 0$,

that has only six integer solutions $\left(\nu_{2 a}, \nu_{2 b}\right)$ as listed in part (iii) of Theorem 2 .

- Let $|u|=3$. By (11) and Proposition 2 we get $\nu_{2 a}+\nu_{2 b}=1$. Again using Proposition 3 we get the system of inequalities:

$$
\begin{aligned}
& \mu_{0}\left(u, \chi_{2}, *\right)=\frac{1}{3}\left(10 \nu_{3 a}-2 \nu_{3 b}+14\right) \geq 0 ; \\
& \mu_{1}\left(u, \chi_{2}, *\right)=\frac{1}{3}\left(-5 \nu_{3 a}+\nu_{3 b}+14\right) \geq 0 ; \\
& \mu_{0}\left(u, \chi_{2}, 2\right)=\frac{1}{3}\left(-6 \nu_{3 a}+6\right) \geq 0,
\end{aligned}
$$

that has only three integer solutions $\left(\nu_{3 a}, \nu_{3 b}\right)$ as listed in part (iv) of Theorem 2

- Let $|u|=4$. By (11) and Proposition 2 we get $\nu_{2 a}+\nu_{2 b}+\nu_{4 a}=1$. We need to consider six cases defined by part (iii) of Theorem 2 In each of these cases, we apply Proposition 3 to get the following systems of inequalities:

$$
\begin{aligned}
\mu_{0}\left(u, \chi_{2}, *\right) & =\frac{1}{4}\left(-4 \nu_{2 a}+4 \nu_{2 b}+4 \nu_{4 a}+\alpha\right) \geq 0 ; \\
\mu_{2}\left(u, \chi_{2}, *\right) & =\frac{1}{4}\left(4 \nu_{2 a}-4 \nu_{2 b}-4 \nu_{4 a}+\alpha\right) \geq 0 ; \\
\mu_{0}\left(u, \chi_{4}, *\right) & =\frac{1}{4}\left(10 \nu_{2 a}-6 \nu_{2 b}+2 \nu_{4 a}+\beta\right) \geq 0 ; \\
\mu_{2}\left(u, \chi_{4}, *\right) & =\frac{1}{4}\left(-10 \nu_{2 a}+6 \nu_{2 b}-2 \nu_{4 a}+\beta\right) \geq 0, \\
\text { where } \quad(\alpha, \beta) & =\left\{\begin{array}{lll}
(12,26) & \text { when } & \chi\left(u^{2}\right)=\chi(2 a) ; \\
(16,18) & \text { when } & \left.\chi u^{2}\right)=\chi(2 b) ; \\
(24,2) & \text { when } & \chi\left(u^{2}\right)=-2 \chi(2 a)+3 \chi(2 b) ; \\
(8,34) & \text { when } & \left.\chi u^{2}\right)=22(2 a)-\chi(2 b) ; \\
(4,42) & \text { when } & \left.\chi u^{2}\right)=3 \chi(2 a)-2 \chi(2 b) ; \\
(20,10) & \text { when } & \chi\left(u^{2}\right)=-\chi(2 a)+2 \chi(2 b) .
\end{array}\right.
\end{aligned}
$$

Additionally, we need to consider the following case-dependent inequalities:

$$
\begin{aligned}
& \mu_{0}\left(u, \chi_{2}, 3\right)=\frac{1}{4}\left(-6 \nu_{2 a}+2 \nu_{2 b}+2 \nu_{4 a}+10\right) \geq 0 \quad \text { for } \chi\left(u^{2}\right)=\chi(2 a) ; \\
& \mu_{0}\left(u, \chi_{8}, *\right)=\frac{1}{4}\left(-20 \nu_{2 a}-4 \nu_{2 b}+4 \nu_{4 a}+68\right) \geq 0 \text { for } \chi\left(u^{2}\right)=\chi(2 b) ; \\
& \mu_{2}\left(u, \chi_{7}, *\right)=\frac{1}{4}\left(-30 \nu_{2 a}+2 \nu_{2 b}-6 \nu_{4 a}+30\right) \geq 0 \text { for } \chi\left(u^{2}\right)=-2 \chi(2 a)+3 \chi(2 b) ; \\
& \mu_{0}\left(u, \chi_{4}, 5\right)=\frac{1}{4}\left(18 \nu_{2 a}+2 \nu_{2 b}+2 \nu_{4 a}+26\right) \geq 0 \quad \text { for } \chi\left(u^{2}\right)=-2 \chi(2 a)+3 \chi(2 b) ; \\
& \mu_{0}\left(u, \chi_{8}, *\right)=\frac{1}{4}\left(-20 \nu_{2 a}-4 \nu_{2 b}+4 \nu_{4 a}+52\right) \geq 0 \quad \text { for } \chi\left(u^{2}\right)=2 \chi(2 a)-\chi(2 b) ; \\
& \mu_{0}\left(u, \chi_{2}, 3\right)=\frac{1}{4}\left(-6 \nu_{2 a}+2 \nu_{2 b}+2 \nu_{4 a}+2\right) \geq 0 \text { for } \chi\left(u^{2}\right)=3 \chi(2 a)-2 \chi(2 b) ; \\
& \mu_{0}\left(u, \chi_{8}, *\right)=\frac{1}{4}\left(-20 \nu_{2 a}-4 \nu_{2 b}+4 \nu_{4 a}+76\right) \geq 0 \text { for } \chi\left(u^{2}\right)=-\chi(2 a)+2 \chi(2 b) ; \\
& \mu_{0}\left(u, \chi_{10}, *\right)=\frac{1}{4}\left(20 \nu_{2 a}+12 \nu_{2 b}-4 \nu_{4 a}+92\right) \geq 0 \text { for } \chi\left(u^{2}\right)=-\chi(2 a)+2 \chi(2 b) .
\end{aligned}
$$

Solving these systems and applying Proposition 5 to the obtained solutions, we get only fifteen integer solutions $\left(\nu_{2 a}, \nu_{2 b}, \nu_{4 a}\right)$ as listed in part (v) of Theorem 2

- Let $|u|=5$. By (11) and Proposition 2 we get $\nu_{5 a}+\nu_{5 b}+\nu_{5 c}+\nu_{5 d}=1$. Put $t=20 \nu_{5 a}+20 \nu_{5 b}$. By Proposition 3 we get the system of inequalities:

$$
\begin{gathered}
\mu_{0}\left(u, \chi_{10}, *\right)=\frac{1}{5}(t+90) \geq 0 ; \quad \mu_{0}\left(u, \chi_{12}, *\right)=\frac{1}{5}(-t+160) \geq 0 \\
\mu_{1}\left(u, \chi_{8}, *\right)=\frac{1}{5}\left(-15 \nu_{5 a}+10 \nu_{5 b}+70\right) \geq 0 \\
\mu_{2}\left(u, \chi_{8}, *\right)=\frac{1}{5}\left(10 \nu_{5 a}-15 \nu_{5 b}+70\right) \geq 0
\end{gathered}
$$


from which we can derive 71 possible pairs $\left(\nu_{5 a}, \nu_{5 b}\right)$. From the inequalities:

$$
\begin{aligned}
& \mu_{0}\left(u, \chi_{7}, 2\right)=\frac{1}{5}\left(16 \nu_{5 a}+16 \nu_{5 b}-4 \nu_{5 c}-4 \nu_{5 d}+64\right) \geq 0 ; \\
& \mu_{0}\left(u, \chi_{6}, 2\right)=\frac{1}{5}\left(-16 \nu_{5 a}-16 \nu_{5 b}+4 \nu_{5 c}+4 \nu_{5 d}+36\right) \geq 0,
\end{aligned}
$$

if follows that $t=4 \nu_{5 a}+4 \nu_{5 b}-\nu_{5 c}-\nu_{5 d} \in\{-16,-11,-6,-1,4,9\}$. Taking into account that $\nu_{5 a}+\nu_{5 b}+\nu_{5 c}+\nu_{5 d}=1$ and considering the additional inequality

$$
\mu_{0}\left(u, \chi_{2}, 2\right)=\frac{1}{5}\left(4 \nu_{5 a}+4 \nu_{5 b}-6 \nu_{5 c}-6 \nu_{5 d}+6\right) \geq 0,
$$

it is easy to check that there remain only 16 possibilities for $\left(\nu_{5 a}, \nu_{5 b}, \nu_{5 c}+\nu_{5 d}\right)$ :

$\{(-2,2,1),(-2,3,0),(-1,1,1),(-1,2,0),(-1,3,-1),(0,0,1),(0,1,0),(0,2,-1)$,

$(1,-1,1),(1,0,0),(1,1,-1),(2,-2,1),(2,-1,0),(2,0,-1),(3,-2,0),(3,-1,-1)\}$.

Finally, using the inequalities

$$
\begin{aligned}
& \mu_{1}\left(u, \chi_{2}, 2\right)=\frac{1}{5}\left(-6 \nu_{5 a}+4 \nu_{5 b}-\nu_{5 c}+4 \nu_{5 d}+6\right) \geq 0 \\
& \mu_{2}\left(u, \chi_{2}, 2\right)=\frac{1}{5}\left(4 \nu_{5 a}-6 \nu_{5 b}+4 \nu_{5 c}-\nu_{5 d}+6\right) \geq 0 \\
& \mu_{1}\left(u, \chi_{4}, 2\right)=\frac{1}{5}\left(-9 \nu_{5 a}+6 \nu_{5 b}+\nu_{5 c}-4 \nu_{5 d}+14\right) \geq 0 \\
& \mu_{2}\left(u, \chi_{4}, 2\right)=\frac{1}{5}\left(6 \nu_{5 a}-9 \nu_{5 b}-4 \nu_{5 c}+\nu_{5 d}+14\right) \geq 0 \\
& \mu_{1}\left(u, \chi_{7}, 2\right)=\frac{1}{5}\left(6 \nu_{5 a}-14 \nu_{5 b}+6 \nu_{5 c}-4 \nu_{5 d}+64\right) \geq 0 \\
& \mu_{2}\left(u, \chi_{7}, 2\right)=\frac{1}{5}\left(-14 \nu_{5 a}+6 \nu_{5 b}-4 \nu_{5 c}+6 \nu_{5 d}+64\right) \geq 0
\end{aligned}
$$

we obtain only ten integer solutions listed in part (vi) of Theorem 2 ,

- Let $|u|=7$. Since there is only one conjugacy class in $G$ consisting of elements or order 7 , this case follows immediately from Proposition 2 ,

- Let $|u|=8$. By (11) and Proposition 2 we get $\nu_{2 a}+\nu_{2 b}+\nu_{4 a}+\nu_{8 a}=1$. Because $\left|u^{2}\right|=4$ and $\left|u^{4}\right|=2$, we need to consider 90 cases defined by parts (iii) and (v) of Theorem 2, First, in 45 of these cases, given in the following table, we have no units of order 8 because $\mu_{1}\left(u, \chi_{2}, *\right)$ is not an integer:

\begin{tabular}{|c|c|c|c|}
\hline$\chi\left(u^{2}\right)$ & $\chi\left(u^{4}\right)=\chi(2 b)$ & $\chi\left(u^{4}\right)=-2 \chi(2 a)+3 \chi(2 b)$ & $\chi\left(u^{4}\right)=2 \chi(2 a)-\chi(2 b)$ \\
\hline$\chi(4 a)$ & & \\
$-2 \chi(2 a)-2 \chi(2 b)+5 \chi(4 a)$ & & & \\
$-\chi(2 a)-3 \chi(2 b)+5 \chi(4 a)$ & & & \\
$-\chi(2 a)-\chi(2 b)+3 \chi(4 a)$ & & & \\
$-\chi(2 a)+\chi(2 b)+\chi(4 a)$ & & & \\
$-4 \chi(2 b)+5 \chi(4 a)$ & & & \\
$-2 \chi(2 b)+3 \chi(4 a)$ & $\mu_{1}\left(u, \chi_{2}, *\right)=\frac{1}{2}$ & \\
$2 \chi(2 b)-\chi(4 a)$ & $\mu_{1}\left(u, \chi_{2}, *\right)=\frac{3}{2}$ & \\
$4 \chi(2 b)-3 \chi(4 a)$ & & \\
$\chi(2 a)-3 \chi(2 b)+3 \chi(4 a)$ & & & \\
$\chi(2 a)-\chi(2 b)+\chi(4 a)$ & & & \\
$\chi(2 a)+\chi(2 b)-\chi(4 a)$ & & & \\
$\chi(2 a)+3 \chi(2 b)-3 \chi(4 a)$ & & & \\
$2 \chi(2 a)-\chi(4 a)$ & & & \\
$2 \chi(2 a)+2 \chi(2 b)-3 \chi(4 a)$ & & & \\
\hline
\end{tabular}

Now put $t_{1}=8 \nu_{2 a}-8 \nu_{2 b}-8 \nu_{4 a}$ and $t_{2}=20 \nu_{2 a}-12 \nu_{2 b}+4 \nu_{4 a}-4 \nu_{8 a}$. Then, when $\chi\left(u^{4}\right)=3 \chi(2 a)-2 \chi(2 b)$ and $\chi\left(u^{2}\right)$ is equal to $2 \chi(2 a)-\chi(4 a)$ or $2 \chi(2 a)+2 \chi(2 b)-$ $3 \chi(4 a)$, we obtain the system of inequalities:

$$
\mu_{0}\left(u, \chi_{2}, *\right)=\frac{1}{8}\left(-t_{1}-8\right) \geq 0 ; \quad \mu_{4}\left(u, \chi_{2}, *\right)=\frac{1}{8}\left(t_{1}-8\right) \geq 0,
$$

which have no integer solutions. Also, there is no solution for the system

$$
\mu_{0}\left(u, \chi_{4}, *\right)=\frac{1}{8}\left(t_{2}+\alpha\right) \geq 0 ; \quad \mu_{4}\left(u, \chi_{4}, *\right)=\frac{1}{8}\left(-t_{2}+\alpha\right) \geq 0,
$$


where $\alpha=-4$ for $\left(\chi\left(u^{4}\right), \chi\left(u^{2}\right)\right)$ in the set

$$
\begin{aligned}
& \{(\chi(2 a), 4 \chi(2 b)-3 \chi(4 a)),(-\chi(2 a)+2 \chi(2 b),-\chi(2 a)+\chi(2 b)+\chi(4 a)), \\
& (-\chi(2 a)+2 \chi(2 b), 2 \chi(2 b)-\chi(4 a)),(-\chi(2 a)+2 \chi(2 b), \chi(2 a)+3 \chi(2 b)-3 \chi(4 a))\}
\end{aligned}
$$

and $\alpha=-20$ for $\left(\chi\left(u^{4}\right), \chi\left(u^{2}\right)\right)=(-\chi(2 a)+2 \chi(2 b), 4 \chi(2 b)-3 \chi(4 a))$.

In the remaining 38 cases, we first consider the following system of inequalities:

$$
\begin{array}{ll}
\mu_{0}\left(u, \chi_{2}, *\right)=\frac{1}{8}\left(-t_{1}+\alpha_{1}\right) \geq 0 ; \quad \mu_{4}\left(u, \chi_{2}, *\right)=\frac{1}{8}\left(t_{1}+\alpha_{1}\right) \geq 0 ; \\
\mu_{0}\left(u, \chi_{4}, *\right)=\frac{1}{8}\left(t_{2}+\alpha_{2}\right) \geq 0 ; \quad \mu_{4}\left(u, \chi_{4}, *\right)=\frac{1}{8}\left(-t_{2}+\alpha_{2}\right) \geq 0 ; \\
\mu_{0}\left(u, \chi_{7}, *\right)=\frac{1}{8}\left(t_{3}+\alpha_{3}\right) \geq 0 ; \quad \mu_{4}\left(u, \chi_{7}, *\right)=\frac{1}{8}\left(-t_{3}+\alpha_{3}\right) \geq 0,
\end{array}
$$

where $t_{3}=60 \nu_{2 a}-4 \nu_{2 b}+12 \nu_{4 a}+4 \nu_{8 a}$ and the tuples $\left(\alpha_{1}, \alpha_{2}, \alpha_{3}\right)$ are given below:

\begin{tabular}{|c|c|c|c|}
\hline & $\chi\left(u^{4}\right)$ & $\chi\left(u^{2}\right)$ & $\left(\alpha_{1}, \alpha_{2}, \alpha_{3}\right)$ \\
\hline 1 & & $\chi(4 a)$ & $(16,28,84)$ \\
2 & & $-2 \chi(2 a)-2 \chi(2 b)+5 \chi(4 a)$ & $(32,28,52)$ \\
3 & & $-\chi(2 a)-3 \chi(2 b)+5 \chi(4 a)$ & $(24,44,84)$ \\
4 & $-\chi(2 a)-\chi(2 b)+3 \chi(4 a)$ & $(24,28,68)$ \\
5 & & $-\chi(2 a)+\chi(2 b)+\chi(4 a)$ & $(24,12,52)$ \\
6 & $-4 \chi(2 b)+5 \chi(4 a)$ & $(16,60,116)$ \\
7 & $\chi(2 a)$ & $-2 \chi(2 b)+3 \chi(4 a)$ & $(16,44,100)$ \\
8 & $2 \chi(2 b)-\chi(4 a)$ & $(16,12,68)$ \\
9 & & $\chi(2 a)-3 \chi(2 b)+3 \chi(4 a)$ & $(8,60,132)$ \\
10 & & $\chi(2 a)-\chi(2 b)+\chi(4 a)$ & $(8,44,116)$ \\
11 & $\chi(2 a)+\chi(2 b)-\chi(4 a)$ & $(8,28,100)$ \\
12 & & $\chi(2 a)+3 \chi(2 b)-3 \chi(4 a)$ & $(8,12,84)$ \\
13 & $2 \chi(2 a)-\chi(4 a)$ & $(0,44,132)$ \\
14 & & $2 \chi(2 a)+2 \chi(2 b)-3 \chi(4 a)$ & $(0,28,116)$ \\
\hline 15 & & $\chi(4 a)$ & $(8,44,116)$ \\
16 & & $-2 \chi(2 a)-2 \chi(2 b)+5 \chi(4 a)$ & $(24,44,84)$ \\
17 & & $-\chi(2 a)-3 \chi(2 b)+5 \chi(4 a)$ & $(16,60,116)$ \\
18 & & $-\chi(2 a)-\chi(2 b)+3 \chi(4 a)$ & $(16,44,100)$ \\
19 & & $-\chi(2 a)+\chi(2 b)+\chi(4 a)$ & $(16,28,84)$ \\
20 & & $-4 \chi(2 b)+5 \chi(4 a)$ & $(8,76,148)$ \\
21 & $3 \chi(2 a)-2 \chi(2 b)$ & $-2 \chi(2 b)+3 \chi(4 a)$ & $(8,60,132)$ \\
22 & $2 \chi(2 b)-\chi(4 a)$ & $(8,28,100)$ \\
23 & & $4 \chi(2 b)-3 \chi(4 a)$ & $(8,12,84)$ \\
24 & & $\chi(2 a)-3 \chi(2 b)+3 \chi(4 a)$ & $(0,76,164)$ \\
25 & & $\chi(2 a)-\chi(2 b)+\chi(4 a)$ & $(0,60,148)$ \\
26 & & $\chi(2 a)+\chi(2 b)-\chi(4 a)$ & $(0,44,132)$ \\
27 & & $\chi(2 a)+3 \chi(2 b)-3 \chi(4 a)$ & $(0,28,116)$ \\
\hline 28 & & $\chi(4 a)$ & $(24,12,52)$ \\
29 & & $-2 \chi(2 a)-2 \chi(2 b)+5 \chi(4 a)$ & $(40,12,20)$ \\
30 & & $-\chi(2 a)-3 \chi(2 b)+5 \chi(4 a)$ & $(32,28,52)$ \\
31 & & $-\chi(2 a)-\chi(2 b)+3 \chi(4 a)$ & $(32,12,36)$ \\
32 & & $-2 \chi(2 b)+5 \chi(4 a)$ & $(24,44,84)$ \\
33 & $-\chi(2 a)+2 \chi(2 b)$ & $(2 a)-3 \chi(4 a)$ & $(24,28,68)$ \\
34 & & $\chi(2 a)-\chi(2 b)+3 \chi(4 a)+\chi(4 a)$ & $(16,44,100)$ \\
35 & & $(16,28,84)$ \\
36 & & $2 \chi(2 a)+2 \chi(2 b)-3 \chi(4 a)-\chi(4 a)$ & $(16,12,68)$ \\
37 & & $(8,28,100)$ \\
38 & & & \\
& & & \\
& & &
\end{tabular}


In all 38 cases these inequalities allow us to compute admissible solutions, using the technique explained in detail in the proof of Theorem 1 in the case of units of order 10. Having done this, we need to consider additional inequalities and apply Proposition 5 to reduce the number of solutions or, possibly, eliminate all of them.

In cases 1, 19 and 35 we use the system of inequalities:

$$
\begin{aligned}
& \mu_{0}\left(u, \chi_{2}, 3\right)=\frac{1}{8}\left(-12 \nu_{2 a}+4 \nu_{2 b}+4 \nu_{4 a}-4 \nu_{8 a}+12\right) \geq 0 ; \\
& \mu_{0}\left(u, \chi_{7}, 3\right)=\frac{1}{8}\left(-28 \nu_{2 a}-12 \nu_{2 b}+4 \nu_{4 a}+4 \nu_{8 a}+52\right) \geq 0 ; \\
& \mu_{4}\left(u, \chi_{7}, 3\right)=\frac{1}{8}\left(28 \nu_{2 a}+12 \nu_{2 b}-4 \nu_{4 a}-4 \nu_{8 a}+52\right) \geq 0,
\end{aligned}
$$

to obtain in all three cases the same set of solutions $\{(0,2,0,-1),(0,-2,2,1)$, $(0,0,2,-1),(0,0,0,1),(-1,-1,2,1),(-1,-1,0,3),(1,1,2,-3),(1,1,0,-1)\}$.

In cases $2,3,4,5,7,16,18,28,30,31,32,33$ and 34 we use the system:

$$
\begin{aligned}
\mu_{4}\left(u, \chi_{10}, *\right) & =\frac{1}{8}\left(-40 \nu_{2 a}-24 \nu_{2 b}+8 \nu_{4 a}+\beta_{1}\right) \geq 0 \\
\mu_{0}\left(u, \chi_{2}, 3\right) & =\frac{1}{8}\left(-12 \nu_{2 a}+4 \nu_{2 b}+4 \nu_{4 a}-4 \nu_{8 a}+\beta_{2}\right) \geq 0 ; \\
\mu_{0}\left(u, \chi_{10}, 7\right) & =\frac{1}{8}\left(36 \nu_{2 a}+20 \nu_{2 b}-12 \nu_{4 a}-4 \nu_{8 a}+\beta_{3}\right) \geq 0,
\end{aligned}
$$

and the following table describes tuples $\left(\beta_{1}, \beta_{2}, \beta_{3}\right)$ and solutions for each case:

\begin{tabular}{|l|c|c|}
\hline Cases & $\left(\beta_{1}, \beta_{2}, \beta_{3}\right)$ & $\left(\nu_{2 a}, \nu_{2 b}, \nu_{4 a}, \nu_{8 a}\right)$ \\
\hline 2,30 & $(16,28,12)$ & $(0,0,-2,3),(0,0,0,1)$ \\
\hline $3,16,32$ & $(24,20,20)$ & $(0,0,2,-1),(0,0,-2,3),(0,0,0,1)$, \\
& & $(1,-1,2,-1),(1,-1,0,1),(-1,1,0,1)$ \\
\hline 4,33 & $(56,20,52)$ & $\begin{array}{r}(0,2,0,-1),(0,-2,0,3),(0,0,2,-1),(0,0,-2,3), \\
(0,0,0,1),(1,-1,0,1),(1,1,2,-3)\end{array}$ \\
\hline 5,28 & $(88,20,84)$ & $(0,-2,0,3),(0,0,2,-1),(0,0,0,1)$, \\
& & $(-1,-1,2,1),(1,1,-2,1),(1,1,0,-1)$ \\
\hline $7,18,34$ & $(64,12,60)$ & $\begin{array}{c}(0,2,-2,1),(0,2,0,-1),(0,0,2,-1),(0,0,0,1), \\
(1,-1,2,-1),(1,1,2,-3),(1,1,0,-1),(-1,1,0,1)\end{array}$ \\
\hline 31 & $(48,28,44)$ & $(0,2,2,-3),(0,0,2,-1),(0,0,0,1)$ \\
\hline
\end{tabular}

In cases 6 and 17 we use the system of inequalities

$$
\begin{aligned}
\mu_{4}\left(u, \chi_{10}, *\right) & =\frac{1}{8}\left(-40 \nu_{2 a}-24 \nu_{2 b}+8 \nu_{4 a}+32\right) \geq 0 ; \\
\mu_{0}\left(u, \chi_{2}, 3\right) & =\frac{1}{8}\left(-12 \nu_{2 a}+4 \nu_{2 b}+4 \nu_{4 a}-4 \nu_{8 a}+12\right) \geq 0 ; \\
\mu_{0}\left(u, \chi_{10}, 7\right) & =\frac{1}{8}\left(36 \nu_{2 a}+20 \nu_{2 b}-12 \nu_{4 a}-4 \nu_{8 a}+28\right) \geq 0 ; \\
\mu_{0}\left(u, \chi_{12}, 7\right) & =\frac{1}{8}\left(-16 \nu_{2 a}+16 \nu_{2 b}-16 \nu_{4 a}+48\right) \geq 0 ; \\
\mu_{4}\left(u, \chi_{12}, 7\right) & =\frac{1}{8}\left(16 \nu_{2 a}-16 \nu_{2 b}+16 \nu_{4 a}+48\right) \geq 0,
\end{aligned}
$$

to obtain in both cases the same set of solutions

$$
\{(0,0,2,-1),(0,0,0,1),(-1,1,0,1)\} .
$$

In cases 8 and 36 we use the system of inequalities:

$$
\begin{aligned}
& \mu_{4}\left(u, \chi_{8}, *\right)=\frac{1}{8}\left(40 \nu_{2 a}+8 \nu_{2 b}-8 \nu_{4 a}+48\right) \geq 0 \\
& \mu_{0}\left(u, \chi_{2}, 3\right)=\frac{1}{8}\left(-12 \nu_{2 a}+4 \nu_{2 b}+4 \nu_{4 a}-4 \nu_{8 a}+12\right) \geq 0 \\
& \mu_{0}\left(u, \chi_{7}, 3\right)=\frac{1}{8}\left(-28 \nu_{2 a}-12 \nu_{2 b}+4 \nu_{4 a}+4 \nu_{8 a}+36\right) \geq 0,
\end{aligned}
$$

to obtain in both cases the same set of solutions $\{(0,0,2,-1),(0,0,0,1)\}$. 
In cases 9,20 and 21 we use the system

$$
\begin{aligned}
\mu_{4}\left(u, \chi_{10}, *\right) & =\frac{1}{8}\left(-40 \nu_{2 a}-24 \nu_{2 b}+8 \nu_{4 a}+\beta_{1}\right) \geq 0 ; \\
\mu_{0}\left(u, \chi_{2}, 3\right) & =\frac{1}{8}\left(-12 \nu_{2 a}+4 \nu_{2 b}+4 \nu_{4 a}-4 \nu_{8 a}+4\right) \geq 0 ; \\
\mu_{0}\left(u, \chi_{10}, 7\right) & =\frac{1}{8}\left(36 \nu_{2 a}+20 \nu_{2 b}-12 \nu_{4 a}-4 \nu_{8 a}+\beta_{2}\right) \geq 0 ; \\
\mu_{0}\left(u, \chi_{12}, 7\right) & =\frac{1}{8}\left(-16 \nu_{2 a}+16 \nu_{2 b}-16 \nu_{4 a}+\beta_{3}\right) \geq 0 ; \\
\mu_{4}\left(u, \chi_{12}, 7\right) & =\frac{1}{8}\left(16 \nu_{2 a}-16 \nu_{2 b}+16 \nu_{4 a}+\beta_{3}\right) \geq 0,
\end{aligned}
$$

where $\left(\beta_{1}, \beta_{2}, \beta_{3}\right)$ is equal to $(72,68,64)$ in cases 9 and 21 , and to $(40,36,32)$ in case 20 . This leads to the solutions

$$
\begin{gathered}
(0,2,-2,1),(0,-2,2,1),(2,0,2,-3),(0,0,0,1), \\
(1,-1,2,-1),(-1,-1,0,3),(-1,1,-2,3),(1,1,0,-1)
\end{gathered}
$$

in cases 9 and 21 and to the unique solution $(0,0,0,1)$ in case 20 .

In cases $10,11,12,15,22,23,37$ and 38 we use the system

$$
\begin{aligned}
& \mu_{0}\left(u, \chi_{8}, *\right)=\frac{1}{8}\left(-40 \nu_{2 a}-8 \nu_{2 b}+8 \nu_{4 a}+\beta_{1}\right) \geq 0 \\
& \mu_{0}\left(u, \chi_{2}, 3\right)=\frac{1}{8}\left(-12 \nu_{2 a}+4 \nu_{2 b}+4 \nu_{4 a}-4 \nu_{8 a}+4\right) \geq 0 \\
& \mu_{4}\left(u, \chi_{7}, 3\right)=\frac{1}{8}\left(28 \nu_{2 a}+12 \nu_{2 b}-4 \nu_{4 a}-4 \nu_{8 a}+\beta_{2}\right) \geq 0,
\end{aligned}
$$

and the following table describes tuples $\left(\beta_{1}, \beta_{2}\right)$ and solutions for each case:

\begin{tabular}{|l|c|c|}
\hline Cases & $\left(\beta_{1}, \beta_{2}\right)$ & $\left(\nu_{2 a}, \nu_{2 b}, \nu_{4 a}, \nu_{8 a}\right)$ \\
\hline 10,15 & $(48,44)$ & $(0,2,-2,1),(0,-2,2,1),(0,0,0,1),(1,-1,2,-1),(1,1,0,-1)$ \\
$11,22,37$ & $(32,28)$ & $(0,0,0,1)$ \\
$12,23,38$ & $(16,12)$ & $(0,0,0,1)$ \\
\hline
\end{tabular}

Finally, in cases $13,14,24,25,26,27$ we use the additional inequality

$$
\mu_{0}\left(u, \chi_{2}, 3\right)=\frac{1}{8}\left(-12 \nu_{2 a}+4 \nu_{2 b}+4 \nu_{4 a}-4 \nu_{8 a}-4\right) \geq 0,
$$

and in the case 29 we use two additional inequalities:

$$
\begin{aligned}
& \mu_{4}\left(u, \chi_{10}, *\right)=\frac{1}{8}\left(-40 \nu_{2 a}-24 \nu_{2 b}+8 \nu_{4 a}+8\right) \geq 0 \\
& \mu_{0}\left(u, \chi_{10}, 7\right)=\frac{1}{8}\left(36 \nu_{2 a}+20 \nu_{2 b}-12 \nu_{4 a}-4 \nu_{8 a}+4\right) \geq 0
\end{aligned}
$$

to show that in these cases we have no solutions.

Now the union of the solutions obtained above gives us part (vii) of Theorem 2 ,

- Let $|u|=15$. By (1) and Proposition 2 we get

$$
\nu_{3 a}+\nu_{3 b}+\nu_{5 a}+\nu_{5 b}+\nu_{5 c}+\nu_{5 d}+\nu_{15 a}+\nu_{15 b}=1
$$

We need to consider 30 cases defined by parts (iv) and (vi) of Theorem 2, Only in two cases we will get a system of inequalities that has solutions. Furthermore, each time these solutions are trivial, so they will give the proof for order 15 . 
First, in eight cases we obtain the following system of inequalities that has no solutions such that all $\mu_{i}\left(u, \chi_{j}, *\right)$ are non-negative integers:

$$
\begin{aligned}
& \mu_{0}\left(u, \chi_{6}, *\right)=\frac{1}{15}\left(72 \nu_{3 a}-32 \nu_{5 a}-32 \nu_{5 b}+8 \nu_{5 c}+8 \nu_{5 d}-8 \nu_{15 a}-8 \nu_{15 b}+\alpha\right) \geq 0 \\
& \mu_{5}\left(u, \chi_{6}, *\right)=\frac{1}{15}\left(-36 \nu_{3 a}+16 \nu_{5 a}+16 \nu_{5 b}-4 \nu_{5 c}-4 \nu_{5 d}+4 \nu_{15 a}+4 \nu_{15 b}+\beta\right) \geq 0,
\end{aligned}
$$

where the tuples $(\alpha, \beta)$ are given in the following table:

\begin{tabular}{|c|c|c|}
\hline$\chi\left(u^{5}\right)$ & $\chi\left(u^{3}\right)$ & $(\alpha, \beta)$ \\
\hline$\chi(3 a)$ & $\chi(5 a)+\chi(5 b)-\chi(5 d)$ & $(18,-9)$ \\
& $\chi(5 a)+\chi(5 b)-\chi(5 c)$ & \\
\hline & $\chi(5 a), \chi(5 b)$ & $(2,29)$ \\
$-\chi(3 a)+2 \chi(3 b)$ & $\chi(5 a)-\chi(5 c)+\chi(5 d)$ & \\
& $\chi(5 b)+\chi(5 c)-\chi(5 d)$ & \\
\hline$-\chi(3 a)+2 \chi(3 b)$ & $\chi(5 a)+\chi(5 b)-\chi(5 d)$ & $(-18,9)$ \\
& $\chi(5 b)+\chi(5 c)-\chi(5 d)$ & \\
\hline
\end{tabular}

In four other cases we obtain the following system of inequalities that has no solution such that all $\mu_{i}\left(u, \chi_{j}, *\right)$ are non-negative integers:

$$
\begin{aligned}
& \mu_{0}\left(u, \chi_{2}, *\right)=\frac{1}{15}\left(40 \nu_{3 a}-8 \nu_{3 b}+12 \nu_{5 a}+12 \nu_{5 b}+12 \nu_{5 c}+12 \nu_{5 d}+6\right) \geq 0 \\
& \mu_{1}\left(u, \chi_{2}, *\right)=\frac{1}{15}\left(5 \nu_{3 a}-\nu_{3 b}-6 \nu_{5 a}+9 \nu_{5 b}+4 \nu_{5 c}-\nu_{5 d}+\alpha_{1}\right) \geq 0 \\
& \mu_{3}\left(u, \chi_{2}, *\right)=\frac{1}{15}\left(-10 \nu_{3 a}+2 \nu_{3 b}-18 \nu_{5 a}+12 \nu_{5 b}+2 \nu_{5 c}-8 \nu_{5 d}+\alpha_{7}\right) \geq 0 \\
& \mu_{5}\left(u, \chi_{2}, *\right)=\frac{1}{15}\left(-20 \nu_{3 a}+4 \nu_{3 b}-6 \nu_{5 a}-6 \nu_{5 b}-6 \nu_{5 c}-6 \nu_{5 d}+27\right) \geq 0 \\
& \mu_{6}\left(u, \chi_{2}, *\right)=\frac{1}{15}\left(-10 \nu_{3 a}+2 \nu_{3 b}+12 \nu_{5 a}-18 \nu_{5 b}-8 \nu_{5 c}+2 \nu_{5 d}+\alpha_{2}\right) \geq 0 \\
& \mu_{0}\left(u, \chi_{4}, *\right)=\frac{1}{15}\left(24 \nu_{3 a}+28 \nu_{5 a}+28 \nu_{5 b}+8 \nu_{5 c}+8 \nu_{5 d}+4 \nu_{15 a}+4 \nu_{15 b}+19\right) \geq 0 \\
& \mu_{1}\left(u, \chi_{4}, *\right)=\frac{1}{15}\left(3 \nu_{3 a}+6 \nu_{5 a}+\nu_{5 b}-4 \nu_{5 c}+6 \nu_{5 d}+3 \nu_{15 a}-2 \nu_{15 b}+\alpha_{3}\right) \geq 0 \\
& \mu_{5}\left(u, \chi_{4}, *\right)=\frac{1}{15}\left(-12 \nu_{3 a}-14 \nu_{5 a}-14 \nu_{5 b}-4 \nu_{5 c}-4 \nu_{5 d}-2 \nu_{15 a}-2 \nu_{15 b}+28\right) \geq 0 \\
& \mu_{6}\left(u, \chi_{4}, *\right)=\frac{1}{15}\left(-6 \nu_{3 a}-12 \nu_{5 a}-2 \nu_{5 b}+8 \nu_{5 c}-12 \nu_{5 d}-6 \nu_{15 a}+4 \nu_{15 b}+\alpha_{4}\right) \geq 0 \\
& \mu_{0}\left(u, \chi_{6}, *\right)=\frac{1}{15}\left(72 \nu_{3 a}-32 \nu_{5 a}-32 \nu_{5 b}+8 \nu_{5 c}+8 \nu_{5 d}-8 \nu_{15 a}-8 \nu_{15 b}+22\right) \geq 0 \\
& \mu_{3}\left(u, \chi_{6}, *\right)=\frac{1}{15}\left(-18 \nu_{3 a}+8 \nu_{5 a}+8 \nu_{5 b}-2 \nu_{5 c}-2 \nu_{5 d}+2 \nu_{15 a}+2 \nu_{15 b}+17\right) \geq 0 \\
& \mu_{0}\left(u, \chi_{11}, *\right)=\frac{1}{15}\left(-72 \nu_{3 a}+8 \nu_{5 a}+8 \nu_{5 b}+8 \nu_{5 c}+8 \nu_{5 d}+8 \nu_{15 a}+8 \nu_{15 b}+148\right) \geq 0 \\
& \mu_{5}\left(u, \chi_{11}, *\right)=\frac{1}{15}\left(36 \nu_{3 a}-4 \nu_{5 a}-4 \nu_{5 b}-4 \nu_{5 c}-4 \nu_{5 d}-4 \nu_{15 a}-4 \nu_{15 b}+121\right) \geq 0 \\
& \mu_{1}\left(u, \chi_{16}, *\right)=\frac{1}{15}\left(8 \nu_{3 a}-\nu_{3 b}+9 \nu_{5 a}-11 \nu_{5 b}+4 \nu_{5 c}-6 \nu_{5 d}+3 \nu_{15 a}-2 \nu_{15 b}+\alpha \alpha_{5}\right) \geq 0 \\
& \mu_{6}\left(u, \chi_{16}, *\right)=\frac{1}{15}\left(-16 \nu_{3 a}+2 \nu_{3 b}-18 \nu_{5 a}+22 \nu_{5 b}-8 \nu_{5 c}\right. \\
&\left.\quad+12 \nu_{5 d}-6 \nu_{15 a}+4 \nu_{15 b}+\alpha_{6}\right) \geq 0
\end{aligned}
$$

where the tuples $\left(\alpha_{1}, \alpha_{2}, \alpha_{3}, \alpha_{4}, \alpha_{5}, \alpha_{6}, \alpha_{7}\right)$ are given in the following table:

\begin{tabular}{|c|c|c|}
\hline$\chi\left(u^{5}\right)$ & $\chi\left(u^{3}\right)$ & $\left(\alpha_{1}, \alpha_{2}, \alpha_{3}, \alpha_{4}, \alpha_{5}, \alpha_{6}, \alpha_{7}\right)$ \\
\hline$-\chi(3 a)+2 \chi(3 b)$ & $\chi(5 c)$ & $(22,1,18,9,240,210,-4)$ \\
\hline$-\chi(3 a)+2 \chi(3 b)$ & $\chi(5 d)$ & $(17,-4,28,19,230,200,1)$ \\
\hline$-\chi(3 a)+2 \chi(3 b)$ & $2 \chi(5 c)-\chi(5 d)$ & $(27,6,8,-1,250,220,-9)$ \\
\hline$-\chi(3 a)+2 \chi(3 b)$ & $-\chi(5 c)+2 \chi(5 d)$ & $(12,-9,38,29,220,190,6)$ \\
\hline
\end{tabular}


In the remaining 18 cases we first consider the following system of inequalities:

$$
\begin{aligned}
& \mu_{0}\left(u, \chi_{2}, *\right)=\frac{1}{15}\left(40 \nu_{3 a}-8 \nu_{3 b}+12 \nu_{5 a}+12 \nu_{5 b}+12 \nu_{5 c}+12 \nu_{5 d}+\alpha_{1}\right) \geq 0 \\
& \mu_{1}\left(u, \chi_{2}, *\right)=\frac{1}{15}\left(5 \nu_{3 a}-\nu_{3 b}-6 \nu_{5 a}+9 \nu_{5 b}+4 \nu_{5 c}-\nu_{5 d}+\alpha_{2}\right) \geq 0 \\
& \mu_{5}\left(u, \chi_{2}, *\right)=\frac{1}{15}\left(-20 \nu_{3 a}+4 \nu_{3 b}-6 \nu_{5 a}-6 \nu_{5 b}-6 \nu_{5 c}-6 \nu_{5 d}+\alpha_{3}\right) \geq 0 \\
& \mu_{6}\left(u, \chi_{2}, *\right)=\frac{1}{15}\left(-10 \nu_{3 a}+2 \nu_{3 b}+12 \nu_{5 a}-18 \nu_{5 b}-8 \nu_{5 c}+2 \nu_{5 d}+\alpha_{4}\right) \geq 0 \\
& \mu_{0}\left(u, \chi_{4}, *\right)=\frac{1}{15}\left(24 \nu_{3 a}+28 \nu_{5 a}+28 \nu_{5 b}+8 \nu_{5 c}+8 \nu_{5 d}+4 \nu_{15 a}+4 \nu_{15 b}+\alpha_{5}\right) \geq 0 \\
& \mu_{1}\left(u, \chi_{4}, *\right)=\frac{1}{15}\left(3 \nu_{3 a}+6 \nu_{5 a}+\nu_{5 b}-4 \nu_{5 c}+6 \nu_{5 d}+3 \nu_{15 a}-2 \nu_{15 b}+\alpha_{6}\right) \geq 0 \\
& \mu_{5}\left(u, \chi_{4}, *\right)=\frac{1}{15}\left(-12 \nu_{3 a}-14 \nu_{5 a}-14 \nu_{5 b}-4 \nu_{5 c}-4 \nu_{5 d}-2 \nu_{15 a}-2 \nu_{15 b}+\alpha_{7}\right) \geq 0 \\
& \mu_{6}\left(u, \chi_{4}, *\right)=\frac{1}{15}\left(-6 \nu_{3 a}-12 \nu_{5 a}-2 \nu_{5 b}+8 \nu_{5 c}-12 \nu_{5 d}-6 \nu_{15 a}+4 \nu_{15 b}+\alpha_{8}\right) \geq 0 \\
& \mu_{0}\left(u, \chi_{6}, *\right)=\frac{1}{15}\left(72 \nu_{3 a}-32 \nu_{5 a}-32 \nu_{5 b}+8 \nu_{5 c}+8 \nu_{5 d}-8 \nu_{15 a}-8 \nu_{15 b}+\alpha_{9}\right) \geq 0 \\
& \mu_{5}\left(u, \chi_{6}, *\right)=\frac{1}{15}\left(-36 \nu_{3 a}+16 \nu_{5 a}+16 \nu_{5 b}-4 \nu_{5 c}-4 \nu_{5 d}+4 \nu_{15 a}+4 \nu_{15 b}+\alpha_{10}\right) \geq 0 \\
& \mu_{0}\left(u, \chi_{11}, *\right)=\frac{1}{15}\left(-72 \nu_{3 a}+8 \nu_{5 a}+8 \nu_{5 b}+8 \nu_{5 c}+8 \nu_{5 d}+8 \nu_{15 a}+8 \nu_{15 b}+\alpha_{11}\right) \geq 0 \\
& \mu_{5}\left(u, \chi_{11}, *\right)=\frac{1}{15}\left(36 \nu_{3 a}-4 \nu_{5 a}-4 \nu_{5 b}-4 \nu_{5 c}-4 \nu_{5 d}-4 \nu_{15 a}-4 \nu_{15 b}+\alpha_{12}\right) \geq 0 \\
& \mu_{1}\left(u, \chi_{16}, *\right)=\frac{1}{15}\left(8 \nu_{3 a}-\nu_{3 b}+9 \nu_{5 a}-11 \nu_{5 b}+4 \nu_{5 c}-6 \nu_{5 d}+3 \nu_{15 a}-2 \nu_{15 b}+\alpha_{13}\right) \geq 0 \\
& \mu_{6}\left(u, \chi_{16}, *\right)=\frac{1}{15}\left(-16 \nu_{3 a}+2 \nu_{3 b}-18 \nu_{5 a}+22 \nu_{5 b}-8 \nu_{5 c}\right. \\
& \left.\quad+12 \nu_{5 d}-6 \nu_{15 a}+4 \nu_{15 b}+\alpha_{14}\right) \geq 0
\end{aligned}
$$

where the tuples $\left(\alpha_{1}, \ldots, \alpha_{14}\right)$ are given in the following table:

\begin{tabular}{|c|c|c|c|}
\hline & $\chi\left(u^{5}\right)$ & $\chi\left(u^{3}\right)$ & $\left(\alpha_{1}, \ldots, \alpha_{14}\right)$ \\
\hline 1 & $\chi(3 a)$ & $\chi(5 a)$ & $(30,0,15,15,41,17,32,26,38,11,112,139,227,251)$ \\
\hline 2 & $\chi(3 a)$ & $\chi(5 b)$ & $(30,15,15,30,41,12,32,21,38,11,112,139,207,231)$ \\
\hline 3 & $\chi(3 a)$ & $\chi(5 c)$ & $(30,10,15,25,31,12,22,21,58,31,112,139,222,246)$ \\
\hline 4 & $\chi(3 a)$ & $\chi(5 d)$ & $(30,5,15,20,31,22,22,31,58,31,112,139,212,236)$ \\
\hline 5 & $\chi(3 b)$ & $\chi(5 a)$ & $(18,6,21,3,35,20,35,20,20,20,130,130,236,233)$ \\
\hline 6 & $\chi(3 b)$ & $\chi(5 b)$ & $(18,21,21,18,35,15,35,15,20,20,130,130,216,213)$ \\
\hline 7 & $\chi(3 b)$ & $\chi(5 c)$ & $(18,16,21,13,25,15,25,15,40,40,130,130,231,228)$ \\
\hline 8 & $\chi(3 b)$ & $\chi(5 d)$ & $(18,11,21,8,25,25,25,25,40,40,130,130,221,218)$ \\
\hline 9 & $\chi(3 a)$ & $2 \chi(5 c)-\chi(5 d)$ & $(30,15,15,30,31,2,22,11,58,31,112,139,232,256)$ \\
\hline 10 & $\chi(3 b)$ & $2 \chi(5 c)-\chi(5 d)$ & $(18,21,21,18,25,5,25,5,40,40,130,130,241,238)$ \\
\hline 11 & $\chi(3 a)$ & $-\chi(5 c)+2 \chi(5 d)$ & $(30,0,15,15,31,32,22,41,58,31,112,139,202,226)$ \\
\hline 12 & $\chi(3 b)$ & $-\chi(5 c)+2 \chi(5 d)$ & $(18,6,21,3,25,35,25,35,40,40,130,130,211,208)$ \\
\hline 13 & $\chi(3 a)$ & $\chi(5 a)-\chi(5 c)+\chi(5 d)$ & $(30,-5,15,10,41,27,32,36,38,11,112,139,217,241)$ \\
\hline 14 & $\chi(3 b)$ & $\chi(5 a)-\chi(5 c)+\chi(5 d)$ & $(18,1,21,-2,35,30,35,30,20,20,130,130,226,223)$ \\
\hline 15 & $\chi(3 b)$ & $\chi(5 a)+\chi(5 b)-\chi(5 d)$ & $(18,16,21,13,45,10,45,10,0,0,130,130,231,228)$ \\
\hline 16 & $\chi(3 b)$ & $\chi(5 a)+\chi(5 b)-\chi(5 c)$ & $(18,11,21,8,45,20,45,20,0,0,130,130,221,218)$ \\
\hline 17 & $\chi(3 a)$ & $\chi(5 b)+\chi(5 c)-\chi(5 d)$ & $(30,20,15,35,41,2,32,11,38,11,112,139,217,241)$ \\
\hline 18 & $\chi(3 b)$ & $\chi(5 b)+\chi(5 c)-\chi(5 d)$ & $(18,26,21,23,35,5,35,5,20,20,130,130,226,223)$ \\
\hline
\end{tabular}

In cases 3, 4, 9 and 11 we use the additional inequalities

$$
\begin{aligned}
& \mu_{0}\left(u, \chi_{2}, 2\right)=\frac{1}{15}\left(-24 \nu_{3 a}+8 \nu_{5 a}+8 \nu_{5 b}-12 \nu_{5 c}-12 \nu_{5 d}-4 \nu_{15 a}-4 \nu_{15 b}-6\right) \geq 0 \\
& \mu_{0}\left(u, \chi_{17}, 7\right)=\frac{1}{15}\left(-64 \nu_{3 a}-16 \nu_{3 b}-8 \nu_{5 a}-8 \nu_{5 b}\right. \\
&\left.\quad-8 \nu_{5 c}-8 \nu_{5 d}+16 \nu_{15 a}+16 \nu_{15 b}+179\right) \geq 0 .
\end{aligned}
$$

In cases $5,6,14,18$ we use the additional inequalities

$$
\begin{aligned}
\mu_{0}\left(u, \chi_{7}, *\right) & =\frac{1}{15}\left(24 \nu_{3 b}+24 \nu_{5 a}+24 \nu_{5 b}-16 \nu_{5 c}-16 \nu_{5 d}+81\right) \geq 0 \\
\mu_{0}\left(u, \chi_{12}, 7\right) & =\frac{1}{15}\left(56 \nu_{3 a}+8 \nu_{3 b}-8 \nu_{5 a}-8 \nu_{5 b}\right. \\
& \left.-8 \nu_{5 c}-8 \nu_{5 d}+16 \nu_{15 a}+16 \nu_{15 b}+122\right) \geq 0 .
\end{aligned}
$$


In cases $7,8,10$, and 12 we use the additional inequalities

$$
\begin{aligned}
& \mu_{0}\left(u, \chi_{11}, 7\right)=\frac{1}{15}\left(-56 \nu_{3 a}+16 \nu_{3 b}+8 \nu_{5 a}+8 \nu_{5 b}\right.\left.+8 \nu_{5 c}+8 \nu_{5 d}-16 \nu_{15 a}-16 \nu_{15 b}+109\right) \geq 0 \\
& \mu_{0}\left(u, \chi_{12}, 7\right)=\frac{1}{15}\left(56 \nu_{3 a}+8 \nu_{3 b}-8 \nu_{5 a}-8 \nu_{5 b}\right. \\
&\left.-8 \nu_{5 c}-8 \nu_{5 d}+16 \nu_{15 a}+16 \nu_{15 b}+122\right) \geq 0 .
\end{aligned}
$$

In case 13 we use the additional inequalities

$$
\begin{gathered}
\mu_{3}\left(u, \chi_{4}, *\right)=\frac{1}{15}\left(-6 \nu_{3 a}-2 \nu_{5 a}-12 \nu_{5 b}-12 \nu_{5 c}+8 \nu_{5 d}+4 \nu_{15 a}-6 \nu_{15 b}+11\right) \geq 0 \\
\mu_{6}\left(u, \chi_{2}, 2\right)=\frac{1}{15}\left(6 \nu_{3 a}+8 \nu_{5 a}-12 \nu_{5 b}+8 \nu_{5 c}-2 \nu_{5 d}-4 \nu_{15 a}+6 \nu_{15 b}-1\right) \geq 0 \\
\mu_{3}\left(u, \chi_{7}, 2\right)=\frac{1}{15}\left(16 \nu_{3 a}+4 \nu_{3 b}+12 \nu_{5 a}-28 \nu_{5 b}\right. \\
\left.+12 \nu_{5 c}-8 \nu_{5 d}+6 \nu_{15 a}-4 \nu_{15 b}+44\right) \geq 0 \\
\mu_{0}\left(u, \chi_{17}, 7\right)=\frac{1}{15}\left(-64 \nu_{3 a}-16 \nu_{3 b}-8 \nu_{5 a}-8 \nu_{5 b}\right. \\
\left.-8 \nu_{5 c}-8 \nu_{5 d}+16 \nu_{15 a}+16 \nu_{15 b}+179\right) \geq 0
\end{gathered}
$$

In case 15 we use the additional inequalities

$$
\begin{aligned}
\mu_{0}\left(u, \chi_{12}, *\right) & =\frac{1}{15}\left(128 \nu_{3 a}+8 \nu_{3 b}-40 \nu_{5 a}-40 \nu_{5 b}+8 \nu_{15 a}+8 \nu_{15 b}+122\right) \geq 0 \\
\mu_{3}\left(u, \chi_{2}, 2\right) & =\frac{1}{15}\left(6 \nu_{3 a}-12 \nu_{5 a}+8 \nu_{5 b}-2 \nu_{5 c}+8 \nu_{5 d}+6 \nu_{15 a}-4 \nu_{15 b}+5\right) \geq 0 ; \\
\mu_{0}\left(u, \chi_{11}, 7\right) & =\frac{1}{15}\left(-56 \nu_{3 a}+16 \nu_{3 b}+8 \nu_{5 a}+8 \nu_{5 b}\right. \\
& \left.+8 \nu_{5 c}+8 \nu_{5 d}-16 \nu_{15 a}-16 \nu_{15 b}+109\right) \geq 0 .
\end{aligned}
$$

In case 16 we use the additional inequalities

$$
\begin{aligned}
& \mu_{0}\left(u, \chi_{12}, *\right)=\frac{1}{15}\left(128 \nu_{3 a}+8 \nu_{3 b}-40 \nu_{5 a}-40 \nu_{5 b}+8 \nu_{15 a}+8 \nu_{15 b}+122\right) \geq 0 \\
& \mu_{0}\left(u, \chi_{11}, 7\right)=\frac{1}{15}\left(-56 \nu_{3 a}+16 \nu_{3 b}+8 \nu_{5 a}+8 \nu_{5 b}\right. \\
&\left.+8 \nu_{5 c}+8 \nu_{5 d}-16 \nu_{15 a}-16 \nu_{15 b}+109\right) \geq 0 .
\end{aligned}
$$

In case 17 we use the additional inequalities

$$
\begin{aligned}
& \mu_{2}\left(u, \chi_{2}, *\right)=\frac{1}{15}\left(5 \nu_{3 a}-\nu_{3 b}+9 \nu_{5 a}-6 \nu_{5 b}-\nu_{5 c}+4 \nu_{5 d}-5\right) \geq 0 \\
& \mu_{3}\left(u, \chi_{2}, 2\right)=\frac{1}{15}\left(6 \nu_{3 a}-12 \nu_{5 a}+8 \nu_{5 b}-2 \nu_{5 c}+8 \nu_{5 d}+6 \nu_{15 a}-4 \nu_{15 b}-1\right) \geq 0 .
\end{aligned}
$$

It follows that in all of these cases we have no integral solutions such that all $\mu_{i}\left(u, \chi_{i}, p\right)$ are non-negative integers.

In case 1 we use the additional inequalities

$$
\begin{aligned}
\mu_{3}\left(u, \chi_{4}, *\right) & =\frac{1}{15}\left(-6 \nu_{3 a}-2 \nu_{5 a}-12 \nu_{5 b}-12 \nu_{5 c}+8 \nu_{5 d}+4 \nu_{15 a}-6 \nu_{15 b}+21\right) \geq 0 \\
\mu_{5}\left(u, \chi_{12}, *\right) & =\frac{1}{15}\left(-64 \nu_{3 a}-4 \nu_{3 b}+20 \nu_{5 a}+20 \nu_{5 b}-4 \nu_{15 a}-4 \nu_{15 b}+124\right) \geq 0 \\
\mu_{6}\left(u, \chi_{2}, 2\right) & =\frac{1}{15}\left(6 \nu_{3 a}+8 \nu_{5 a}-12 \nu_{5 b}+8 \nu_{5 c}-2 \nu_{5 d}-4 \nu_{15 a}+6 \nu_{15 b}-6\right) \geq 0 \\
\mu_{3}\left(u, \chi_{7}, 2\right)=\frac{1}{15}\left(16 \nu_{3 a}+4 \nu_{3 b}+12 \nu_{5 a}-28 \nu_{5 b}\right. & \left.+12 \nu_{5 c}-8 \nu_{5 d}+6 \nu_{15 a}-4 \nu_{15 b}+34\right) \geq 0 \\
\mu_{6}\left(u, \chi_{7}, 2\right)=\frac{1}{15}\left(16 \nu_{3 a}+4 \nu_{3 b}-28 \nu_{5 a}+12 \nu_{5 b}\right. & \left.-8 \nu_{5 c}+12 \nu_{5 d}-4 \nu_{15 a}+6 \nu_{15 b}+54\right) \geq 0 \\
\mu_{0}\left(u, \chi_{17}, 7\right)=\frac{1}{15}\left(-64 \nu_{3 a}-16 \nu_{3 b}-8 \nu_{5 a}-8 \nu_{5 b}\right. & \left.-8 \nu_{5 c}-8 \nu_{5 d}+16 \nu_{15 a}+16 \nu_{15 b}+179\right) \geq 0
\end{aligned}
$$

to obtain only one trivial solution with $\nu_{15 b}=1$. 
In case 2 we will use the additional inequalities

$$
\begin{aligned}
\mu_{2}\left(u, \chi_{2}, *\right) & =\frac{1}{15}\left(5 \nu_{3 a}-\nu_{3 b}+9 \nu_{5 a}-6 \nu_{5 b}-\nu_{5 c}+4 \nu_{5 d}\right) \geq 0 \\
\mu_{5}\left(u, \chi_{12}, *\right) & =\frac{1}{15}\left(-64 \nu_{3 a}-4 \nu_{3 b}+20 \nu_{5 a}+20 \nu_{5 b}-4 \nu_{15 a}-4 \nu_{15 b}+124\right) \geq 0 \\
\mu_{3}\left(u, \chi_{2}, 2\right) & =\frac{1}{15}\left(6 \nu_{3 a}-12 \nu_{5 a}+8 \nu_{5 b}-2 \nu_{5 c}+8 \nu_{5 d}+6 \nu_{15 a}-4 \nu_{15 b}-6\right) \geq 0 \\
\mu_{3}\left(u, \chi_{7}, 2\right)=\frac{1}{15}\left(16 \nu_{3 a}+4 \nu_{3 b}+12 \nu_{5 a}-28 \nu_{5 b}\right. & \left.+12 \nu_{5 c}-8 \nu_{5 d}+6 \nu_{15 a}-4 \nu_{15 b}+54\right) \geq 0 \\
\mu_{6}\left(u, \chi_{7}, 2\right)=\frac{1}{15}\left(16 \nu_{3 a}+4 \nu_{3 b}-28 \nu_{5 a}+12 \nu_{5 b}\right. & \left.-8 \nu_{5 c}+12 \nu_{5 d}-4 \nu_{15 a}+6 \nu_{15 b}+34\right) \geq 0 \\
\mu_{0}\left(u, \chi_{17}, 7\right)=\frac{1}{15}\left(-64 \nu_{3 a}-16 \nu_{3 b}-8 \nu_{5 a}-8 \nu_{5 b}\right. & \left.-8 \nu_{5 c}-8 \nu_{5 d}+16 \nu_{15 a}+16 \nu_{15 b}+179\right) \geq 0
\end{aligned}
$$

to show that it has the unique trivial solution with $\nu_{15 a}=1$.

- It remains to prove part (i) of Theorem 2, showing that there are no elements of orders 14, 21 and 35 in $V(\mathbb{Z} G)$. As in the proof of Theorem 1 below we give the table containing the data describing the constraints on partial augmentations $\nu_{p}$ and $\nu_{q}$ accordingly to (2)-(4) for all of these orders. From this table part (i) of Theorem 2 is derived in the same way as in the proof of Theorem 1 .

\begin{tabular}{|c|c|c|c|c|c|c|c|c|c|}
\hline$|u|$ & $p$ & $q$ & $\xi, \tau$ & $\xi\left(C_{p}\right)$ & $\xi\left(C_{q}\right)$ & $l$ & $m_{1}$ & $m_{p}$ & $m_{q}$ \\
\hline & & & & & & 0 & 86 & -30 & 0 \\
14 & 2 & 7 & $\xi=(4,8)_{[*]}$ & -5 & 0 & 2 & 86 & 5 & 0 \\
& & & & & & 7 & 96 & 30 & 0 \\
\hline \multirow{2}{*}{21} & \multirow{2}{*}{3} & \multirow{2}{*}{7} & $\xi=(4,7)_{[*]}$ & 3 & 0 & 0 & 90 & 36 & 0 \\
& & & $\tau=(4,7)_{[*]}$ & 3 & 0 & 7 & 81 & -18 & 0 \\
\hline 35 & 5 & 7 & $\xi=(2,19)_{[*]}$ & 0 & 2 & 1 & 511 & 0 & 2 \\
\hline & & & & 3 & 0 & 0 & 40 & 72 & 0 \\
{$[* *$} & & 3 & 0 & 7 & 25 & -18 & 0 \\
\hline
\end{tabular}

\section{Proof of Theorem 3}

Let $G$ be the third Janko simple group $J_{3}$. It is well known [13, 15] that $|G|=$ $2^{7} \cdot 3^{5} \cdot 5 \cdot 17 \cdot 19$ and $\exp (G)=2^{3} \cdot 3^{2} \cdot 5 \cdot 17 \cdot 19$.

The group $G$ only possesses elements of orders $2,3,4,5,6,8,9,10,12,15$, 17 and 19. Hence, we shall first investigate normalized units of these orders. By Proposition 4, the order of each torsion unit divides the exponent of $G$. So, second we consider normalized units of orders $18,20,24,30,34,38,45,51,57,85,95$ and 323. We shall prove that units of all these orders except 18,20, 24, 30 and 45 do not appear in $V(\mathbb{Z} G)$. We will omit cases of units of orders 18, 20, 24, 30 and 45 since they are not products of two disctinct primes, so they do not contribute to Kimmerle's conjecture.

Assume that $u$ is a non-trivial normalized unit and consider each case separately.

- Let $|u|=2$. Since there is only one conjugacy class in $G$ consisting of elements or order 2, this case immediately follows from Propositions 11 and 2 ,

- Let $|u|=3$. By (11) and Proposition 2 we have $\nu_{3 a}+\nu_{3 b}=1$. By Proposition 3

$$
\begin{aligned}
& \mu_{0}\left(u, \chi_{2}, *\right)=\frac{1}{3}\left(-10 \nu_{3 a}+8 \nu_{3 b}+85\right) \geq 0 \\
& \mu_{1}\left(u, \chi_{2}, *\right)=\frac{1}{3}\left(5 \nu_{3 a}-4 \nu_{3 b}+85\right) \geq 0 \\
& \mu_{0}\left(u, \chi_{4}, 2\right)=\frac{1}{3}\left(16 \nu_{3 a}-2 \nu_{3 b}+80\right) \geq 0
\end{aligned}
$$


and this system only has the ten solutions listed in part (iii) of Theorem 3 ,

- Let $|u|=4$. By (11) and Proposition 2 we have $\nu_{2 a}+\nu_{4 a}=1$. By Proposition 3

$$
\begin{array}{ll}
\mu_{0}\left(u, \chi_{2}, *\right)=\frac{1}{4}\left(t_{1}+90\right) \geq 0 ; & \mu_{2}\left(u, \chi_{2}, *\right)=\frac{1}{4}\left(-t_{1}+90\right) \geq 0 ; \\
\mu_{0}\left(u, \chi_{2}, 3\right)=\frac{1}{4}\left(t_{2}+20\right) \geq 0 ; & \mu_{2}\left(u, \chi_{2}, 3\right)=\frac{1}{4}\left(-t_{2}+20\right) \geq 0,
\end{array}
$$

where $t_{1}=10 \nu_{2 a}+2 \nu_{4 a}$ and $t_{2}=4 \nu_{2 a}-4 \nu_{4 a}$. Solving this system and applying Proposition [5] only the three solutions listed in part (iv) of Theorem 3 remain.

- Let $|u|=5$. Then $\nu_{5 a}+\nu_{5 b}=1$, and we have the system

$$
\begin{aligned}
& \mu_{1}\left(u, \chi_{8}, 2\right)=\frac{1}{5}\left(3 \nu_{5 a}-2 \nu_{5 b}+322\right) \geq 0 ; \\
& \mu_{1}\left(u, \chi_{2}, 3\right)=\frac{1}{5}\left(-3 \nu_{5 a}+2 \nu_{5 b}+18\right) \geq 0 ; \\
& \mu_{2}\left(u, \chi_{2}, 3\right)=\frac{1}{5}\left(2 \nu_{5 a}-3 \nu_{5 b}+18\right) \geq 0,
\end{aligned}
$$

which only has the eight solutions listed in part (v) of Theorem 3

- Let $|u|=8$. Then $\nu_{2 a}+\nu_{4 a}+\nu_{8 a}=1$ and we need to consider three cases defined by part (iv) of Theorem 3 . For all of them, put $t_{1}=20 \nu_{2 a}+4 \nu_{4 a}-4 \nu_{8 a}$, $t_{2}=12 \nu_{2 a}+12 \nu_{4 a}-4 \nu_{8 a}$ and $t_{3}=8 \nu_{2 a}-8 \nu_{4 a}$.

Case 1. $\chi\left(u^{2}\right)=\chi(4 a)$. We have the system of inequalities

$$
\begin{gathered}
\mu_{0}\left(u, \chi_{2}, *\right)=\frac{1}{8}\left(t_{1}+92\right) \geq 0 ; \quad \mu_{4}\left(u, \chi_{2}, *\right)=\frac{1}{8}\left(-t_{1}+92\right) \geq 0 ; \\
\mu_{0}\left(u, \chi_{4}, *\right)=\frac{1}{8}\left(t_{2}+332\right) \geq 0 ; \quad \mu_{4}\left(u, \chi_{4}, *\right)=\frac{1}{8}\left(-t_{2}+332\right) \geq 0 ; \\
\mu_{0}\left(u, \chi_{2}, 3\right)=\frac{1}{8}\left(t_{3}+16\right) \geq 0 ; \quad \mu_{4}\left(u, \chi_{2}, 3\right)=\frac{1}{8}\left(-t_{3}+16\right) \geq 0 ; \\
\mu_{0}\left(u, \chi_{4}, 3\right)=\frac{1}{8}\left(16 \nu_{2 a}-8 \nu_{8 a}+88\right) \geq 0,
\end{gathered}
$$

which only has the following nine solutions satisfying Proposition 5 such that all $\mu_{i}\left(u, \chi_{j}, p\right)$ are non-negative integers:

$$
\begin{aligned}
&\left(\nu_{2 a}, \nu_{4 a}, \nu_{8 a}\right) \in\{(2,4,-5),(2,0,-1),(-2,0,3),(0,0,1),(0,2,-1), \\
&(2,2,-3),(-2,-4,7),(-2,-2,5),(0,-2,3)\} .
\end{aligned}
$$

Case 2. $\chi\left(u^{2}\right)=-2 \chi(2 a)+3 \chi(4 a)$. Then we obtain the system

$$
\begin{array}{ll}
\mu_{0}\left(u, \chi_{2}, *\right)=\frac{1}{8}\left(t_{1}+76\right) \geq 0 ; & \mu_{4}\left(u, \chi_{2}, *\right)=\frac{1}{8}\left(-t_{1}+76\right) \geq 0 ; \\
\mu_{0}\left(u, \chi_{4}, *\right)=\frac{1}{8}\left(t_{2}+332\right) \geq 0 ; & \mu_{4}\left(u, \chi_{4}, *\right)=\frac{1}{8}\left(-t_{2}+332\right) \geq 0 ; \\
\mu_{0}\left(u, \chi_{2}, 3\right)=\frac{1}{8} t_{3} \geq 0 ; & \mu_{4}\left(u, \chi_{2}, 3\right)=\frac{1}{8}\left(-t_{3}\right) \geq 0,
\end{array}
$$

which has only three solutions satisfying Proposition 5 such that all $\mu_{i}\left(u, \chi_{j}, p\right)$ are non-negative integers: $\left(\nu_{2 a}, \nu_{4 a}, \nu_{8 a}\right) \in\{(0,0,1),(-2,-2,5),(2,2,-3)\}$.

Case 3. $\chi\left(u^{2}\right)=2 \chi(2 a)-\chi(4 a)$. Put $t_{4}=28 \nu_{2 a}-4 \nu_{4 a}-4 \nu_{8 a}$. Then the system

$$
\begin{gathered}
\mu_{0}\left(u, \chi_{2}, *\right)=\frac{1}{8}\left(t_{1}+108\right) \geq 0 ; \quad \mu_{4}\left(u, \chi_{2}, *\right)=\frac{1}{8}\left(-t_{1}+108\right) \geq 0 ; \\
\mu_{0}\left(u, \chi_{4}, *\right)=\frac{1}{8}\left(t_{2}+332\right) \geq 0 ; \quad \mu_{4}\left(u, \chi_{4}, *\right)=\frac{1}{8}\left(-t_{2}+332\right) \geq 0 ; \\
\mu_{0}\left(u, \chi_{2}, 3\right)=\frac{1}{8}\left(t_{3}+32\right) \geq 0 ; \quad \mu_{4}\left(u, \chi_{2}, 3\right)=\frac{1}{8}\left(-t_{3}+32\right) \geq 0 ; \\
\mu_{0}\left(u, \chi_{6}, 3\right)=\frac{1}{8}\left(-t_{4}+116\right) \geq 0 ; \quad \mu_{4}\left(u, \chi_{6}, 3\right)=\frac{1}{8}\left(t_{4}+116\right) \geq 0 ; \\
\mu_{0}\left(u, \chi_{4}, 3\right)=\frac{1}{8}\left(16 \nu_{2 a}-8 \nu_{8 a}+104\right) \geq 0,
\end{gathered}
$$


only has the following nine solutions satisfying Proposition [5 such that all $\mu_{i}\left(u, \chi_{j}, p\right)$ are non-negative integers:

$$
\begin{aligned}
\left(\nu_{2 a}, \nu_{4 a}, \nu_{8 a}\right) \in\{ & (2,6,-7),(2,4,-5),(0,4,-3),(2,0,-1),(-2,0,3), \\
& (0,0,1),(-2,-6,9),(0,2,-1),(2,2,-3),(-2,2,1), \\
& (-2,-4,7),(0,-4,5),(-2,-2,5),(0,-2,3),(2,-2,1)\} .
\end{aligned}
$$

The union of the solutions of all three cases gives us part (vi) of Theorem 3 .

- Let $|u|=17$. Then $\nu_{17 a}+\nu_{17 b}=1$ and we have the system

$$
\begin{gathered}
\mu_{1}\left(u, \chi_{3}, 19\right)=\frac{1}{17}(t+110) \geq 0 ; \quad \mu_{1}\left(u, \chi_{9}, 19\right)=\frac{1}{17}(-t+706) \geq 0 ; \\
\mu_{1}\left(u, \chi_{2}, 2\right)=\frac{1}{17}\left(-10 \nu_{17 a}+7 \nu_{17 b}+78\right) \geq 0 ; \\
\mu_{3}\left(u, \chi_{2}, 2\right)=\frac{1}{17}\left(7 \nu_{17 a}-10 \nu_{17 b}+78\right) \geq 0,
\end{gathered}
$$

where $t=9 \nu_{17 a}-8 \nu_{17 b}$, with the ten solutions listed in part (vii) of Theorem 3

- Let $|u|=19$. Then $\nu_{19 a}+\nu_{19 b}=1$, and we have the system

\begin{tabular}{|c|c|c|c|c|c|c|c|c|c|}
\hline$|u|$ & $p$ & $q$ & $\xi, \tau$ & $\xi\left(C_{p}\right)$ & $\xi\left(C_{q}\right)$ & $l$ & $m_{1}$ & $m_{p}$ & $m_{q}$ \\
\hline \multirow{3}{*}{34} & \multirow{3}{*}{2} & \multirow{3}{*}{17} & \multirow{3}{*}{$\xi=(2)_{[*]}$} & \multirow{3}{*}{5} & \multirow{3}{*}{0} & 0 & 90 & 80 & 0 \\
\hline & & & & & & 1 & 80 & 5 & 0 \\
\hline & & & & & & 17 & 80 & -80 & 0 \\
\hline \multirow{3}{*}{38} & \multirow{3}{*}{2} & \multirow{3}{*}{18} & $\xi=(7)_{[*]}$ & -10 & 0 & 0 & 636 & -180 & 0 \\
\hline & & & $\xi=(7)_{[*]}$ & -10 & 0 & 19 & 656 & 180 & 0 \\
\hline & & & $\tau=(13)_{[*]}$ & 15 & 0 & 1 & 1600 & 15 & 0 \\
\hline \multirow{3}{*}{51} & \multirow{3}{*}{3} & \multirow{3}{*}{17} & $\xi=(9)_{[*]}$ & 6 & 0 & 0 & 828 & 192 & 0 \\
\hline & & & $\xi=(9)_{[*]}$ & 6 & 0 & 17 & 810 & -96 & 0 \\
\hline & & & $\tau=(13)_{[*]}$ & -5 & 0 & 0 & 1605 & -160 & 0 \\
\hline \multirow{3}{*}{57} & \multirow{3}{*}{3} & \multirow{3}{*}{19} & \multirow{3}{*}{$\xi=(13)_{[*]}$} & \multirow{3}{*}{-5} & \multirow{3}{*}{0} & 0 & 1605 & -180 & 0 \\
\hline & & & & & & 3 & 1605 & 10 & 0 \\
\hline & & & & & & 19 & 1620 & 90 & 0 \\
\hline \multirow[t]{2}{*}{85} & \multirow[t]{2}{*}{5} & \multirow[t]{2}{*}{17} & \multirow[t]{2}{*}{$\xi=(4,8)_{[3]}$} & \multirow[t]{2}{*}{-2} & \multirow[t]{2}{*}{0} & 0 & 400 & $\begin{array}{l}-128 \\
\end{array}$ & 0 \\
\hline & & & & & & 17 & 410 & 32 & 0 \\
\hline \multirow[t]{2}{*}{95} & \multirow[t]{2}{*}{5} & \multirow[t]{2}{*}{19} & \multirow[t]{2}{*}{$\xi=(4)_{[2]}$} & \multirow[t]{2}{*}{0} & \multirow[t]{2}{*}{4} & 0 & 152 & 0 & 288 \\
\hline & & & & & & 19 & 152 & 0 & -72 \\
\hline \multirow[t]{2}{*}{323} & \multirow[t]{2}{*}{17} & \multirow[t]{2}{*}{19} & \multirow[t]{2}{*}{$\xi=(6)_{[3]}$} & \multirow[t]{2}{*}{0} & 1 & 0 & 171 & 0 & 288 \\
\hline & & & & & & 19 & 171 & 0 & -18 \\
\hline
\end{tabular}

$$
\begin{aligned}
& \mu_{1}\left(u, \chi_{2}, *\right)=\frac{1}{19}\left(10 \nu_{19 a}-9 \nu_{19 b}+85\right) \geq 0 \\
& \mu_{2}\left(u, \chi_{2}, *\right)=\frac{1}{19}\left(-9 \nu_{19 a}+10 \nu_{19 b}+85\right) \geq 0 \\
& \mu_{1}\left(u, \chi_{5}, 2\right)=\frac{1}{19}\left(11 \nu_{19 a}-8 \nu_{19 b}+84\right) \geq 0 \\
& \mu_{2}\left(u, \chi_{5}, 2\right)=\frac{1}{19}\left(-8 \nu_{19 a}+11 \nu_{19 b}+84\right) \geq 0
\end{aligned}
$$

with the ten solutions listed in part (viii) of Theorem 3 .

- It remains to prove that there are no elements of orders $34,38,51,57,85,95$ and 323 in $V(\mathbb{Z} G)$. We give the table with the data needed to derive part (i) of Theorem 3 in the same way as in the proofs of Theorem 1 and Theorem 2 .

6. Some REMARKS ON THE GROUP $J_{4}$

If $G \cong J_{4}$, then $|G|=2^{21} \cdot 3^{3} \cdot 5 \cdot 7 \cdot 11^{3} \cdot 23 \cdot 29 \cdot 31 \cdot 37 \cdot 43$ and $\exp (G)=$ $2^{4} \cdot 3 \cdot 5 \cdot 7 \cdot 11 \cdot 23 \cdot 29 \cdot 31 \cdot 37 \cdot 43$ (see [13, 15]). From Propositions 1 and 2 it immediately follows that units of orders $3,5,23$ and 29 are rationally conjugate to a group element. Nevertheless, further computations are limited by the fact 
that $p$-Brauer character tables for $J_{4}$ are not known for $p \in\{2,3,11,23,29,31,43\}$ (see http://www.math.rwth-aachen.de/ ${ }^{\sim} \mathrm{MOC} /$ work.html). For example, for units of order 31 the best restriction that can be obtained by applying Proposition 3 to all known ordinary and $p$-Brauer character tables is given by the system

$$
\begin{aligned}
& \mu_{1}\left(u, \chi_{56}, *\right)=\frac{1}{31}\left(21 \nu_{31 a}-10 \nu_{31 b}-10 \nu_{31 c}+2001151845\right) \geq 0 \\
& \mu_{3}\left(u, \chi_{56}, *\right)=\frac{1}{31}\left(-10 \nu_{31 a}-10 \nu_{31 b}+21 \nu_{31 c}+2001151845\right) \geq 0 \\
& \mu_{5}\left(u, \chi_{56}, *\right)=\frac{1}{31}\left(-10 \nu_{31 a}+21 \nu_{31 b}-10 \nu_{31 c}+2001151845\right) \geq 0
\end{aligned}
$$

where $\nu_{31 a}+\nu_{31 b}+\nu_{31 c}=1$, and all $\mu_{i}\left(u, \chi_{j}, *\right)$ are non-negative integers for every tuple $\left(\nu_{31 a}, \nu_{31 b}, \nu_{31 c}\right)$ such that $\nu_{31 b}, \nu_{31 c} \geq-64553285$ and $\nu_{31 b}+\nu_{31 c} \leq 64553286$ (thus, it will have 18,752,070,203,460,153 solutions, which is too far from rational conjugacy).

Hopefully, further progress can be made using the Luthar-Passi method if $p$ Brauer character tables for the missing values of $p$ will become available.

\section{ACKNOWLEDGMENTS}

The authors are grateful to Ian Gent, Tom Kelsey and Andrea Rendl for their advice in using constraint programming solvers.

\section{REFERENCES}

1. V. A. Artamonov and A. A. Bovdi, Integral group rings: groups of invertible elements and classical K-theory, Algebra. Topology. Geometry, Vol. 27 (Russian), Itogi Nauki i Tekhniki, Akad. Nauk SSSR Vsesoyuz. Inst. Nauchn. i Tekhn. Inform., Moscow, 1989, pp. 3-43, 232. MR.1039822 (91e:16028)

2. V. Bovdi, A. Grishkov, and A. Konovalov, Kimmerle conjecture for the Held and O'Nan sporadic simple groups, Sci. Math. Jpn. 69 (2009), no. 3, 353-361. MR2510100

3. V. Bovdi and M. Hertweck, Zassenhaus conjecture for central extensions of $S_{5}$, J. Group Theory 11 (2008), no. 1, 63-74. MR2381018

4. V. Bovdi and A. Konovalov, Integral group ring of the first Mathieu simple group, Groups St. Andrews 2005. Vol. 1, London Math. Soc. Lecture Note Ser., vol. 339, Cambridge Univ. Press, Cambridge, 2007, pp. 237-245. MR2328163

5. - Integral group ring of the McLaughlin simple group, Algebra Discrete Math. (2007), no. 2, 43-53. MR2364062

6. Integral group ring of the Mathieu simple group $M_{23}$, Comm. Algebra 36 (2008), no. 7, 2670-2680. MR2422512

7. _ Torsion units in integral group ring of Higman-Sims simple group, Studia Sci. Math. Hungar. (to appear, 2009).

8. V. Bovdi, A. Konovalov, and S. Linton, Torsion units in integral group ring of the Mathieu simple group $M_{22}$, LMS J. Comput. Math. 11 (2008), 28-39. MR.2379938

9. V. Bovdi, A. Konovalov, and E.N. Marcos, Integral group ring of the Suzuki sporadic simple group, Publ. Math. Debrecen 72 (2008), no. 3-4, 487-503. MR2406705

10. V. Bovdi, A. Konovalov, R. Rossmanith, and Cs. Schneider, LAGUNA-Lie AlGebras and UNits of group Algebras, Version 3.5.0, 2009, (http://www.cs.st-andrews.ac.uk/ alexk/laguna.htm).

11. V. Bovdi, A. Konovalov, and S. Siciliano, Integral group ring of the Mathieu simple group $M_{12}$, Rend. Circ. Mat. Palermo (2) 56 (2007), no. 1, 125-136. MR2313777

12. J.A. Cohn and D. Livingstone, On the structure of group algebras. I, Canad. J. Math. 17 (1965), 583-593. MR0179266(31:3514)

13. J. H. Conway, R. T. Curtis, S. P. Norton, R. A. Parker, and R. A. Wilson, Atlas of Finite Groups, Oxford University Press, Eynsham, 1985, Maximal subgroups and ordinary characters for simple groups, With computational assistance from J. G. Thackray. MR 827219 (88g:20025)

14. ECLiPSe Constraint Programming System, Ver. 5.10, (http://www.eclipse-clp.org), 2006. 
15. GAP-Groups, Algorithms, and Programming, Version 4.4.12, (http://www.gap-system. org), 2008.

16. I. P. Gent, C. Jefferson, and I. Miguel, Minion: A fast scalable constraint solver, ECAI 2006, 17th European Conference on Artificial Intelligence, August 29 - September 1, 2006, Riva del Garda, Italy, Including Prestigious Applications of Intelligent Systems (PAIS 2006), Proceedings, IOS Press, 2006, pp. 98-102.

17. M. Hertweck, On the torsion units of some integral group rings, Algebra Colloq. 13 (2006), no. 2, 329-348. MR.2208368

18. - The orders of torsion units in integral group rings of finite solvable groups, Comm. Algebra 36 (2008), no. 10, 3585-3588. MR2458394

19. Math. Soc. 51 (2008), no. 2, 363-385. MR2465913 (2009j:16027)

20. - Partial augmentations and Brauer character values of torsion units in group rings, Comm. Algebra (to appear, 2007), 1-16, (E-print arXiv:math.RA/0612429v2).

21. C. Jansen, K. Lux, R. Parker, and R. Wilson, An Atlas of Brauer Characters, London Mathematical Society Monographs. New Series, vol. 11, Clarendon Press Oxford University Press, New York, 1995, Appendix 2 by T. Breuer and S. Norton, Oxford Science Publications. MR $1367961(96 \mathrm{k}: 20016)$

22. W. Kimmerle, On the prime graph of the unit group of integral group rings of finite groups, Groups, rings and algebras, Contemp. Math., vol. 420, Amer. Math. Soc., Providence, RI, 2006, pp. 215-228. MR 2279241

23. I. S. Luthar and I. B. S. Passi, Zassenhaus conjecture for $A_{5}$, Proc. Indian Acad. Sci. Math. Sci. 99 (1989), no. 1, 1-5. MR1004634 (90g:20007)

24. Mini-Workshop: Arithmetik von Gruppenringen, Oberwolfach Rep. 4 (2007), no. 4, 32093239, Abstracts from the mini-workshop held November 25-December 1, 2007, Organized by E. Jespers, Z. Marciniak, G. Nebe and W. Kimmerle, Oberwolfach Reports. Vol. 4, no. 4. MR2463649

25. H. Zassenhaus, On the torsion units of finite group rings, Studies in mathematics (in honor of A. Almeida Costa) (Portuguese), Instituto de Alta Cultura, Lisbon, 1974, pp. 119-126. MR0376747 (51:12922)

Institute of Mathematics, University of Debrecen, P.O. Box 12, H-4010 Debrecen, HuNGARY

E-mail address: vbovdi@math.unideb.hu

Department of Mathematics, Vrije Universiteit Brussel, Pleinlaan 2, B-1050 Brussel, BELGIUM

E-mail address: ef jesper@vub.ac.be

School of Computer Science, University of St Andrews, Jack Cole Building, North Haugh, St Andrews, Fife, KY16 9SX, Scotland

E-mail address: alexk@mcs.st-andrews.ac.uk 